\title{
ON THE BV QUANTIZATION OF GAUGE GRAVITATION THEORY
}

\author{
D.BASHKIROV \\ Department of Theoretical Physics, Moscow State University, 117234, Moscow, Russia \\ bashkir@phys.msu.ru \\ G.SARDANASHVILY \\ Department of Theoretical Physics, Moscow State University, 117234, Moscow, Russia \\ sard@grav.phys.msu.su
}

\begin{abstract}
Quantization of gravitation theory as gauge theory of general covariant transformations in the framework of Batalin-Vilkoviski (BV) formalism is considered. Its gauge-fixed Lagrangian is constructed.
\end{abstract}

\section{Introduction}

Classical gauge gravitation theory has been developed in different variants since 60th (see $[1,2,3]$ for a survey). We describe gravitation theory as a standard gauge theory on fiber bundles where parameters of gauge transformations are vector fields on a base manifold $X$. The well-known BV quantization technique $[4,5]$ can be applied to this theory. Since this quantization technique fails to provide a functional measure, we restrict ourselves to constructing a gauge-fixed Lagrangian.

Recall that an $r$-order Lagrangian of a Lagrangian system on a smooth fiber bundle $Y \rightarrow X$ is defined as a density

$$
L=\mathcal{L} \omega: J^{r} Y \rightarrow \wedge \wedge^{n} T^{*} X, \quad \omega=d x^{1} \wedge \cdots \wedge d x^{n}, \quad n=\operatorname{dim} X,
$$

on the $r$-order jet manifold $J^{r} Y$ of sections of $Y \rightarrow X[6,7]$. Given bundle coordinates $\left(x^{\lambda}, y^{i}\right)$ on $Y$, the jet manifold $J^{r} Y$ is endowed with the adapted coordinates $\left(x^{\lambda}, y^{i}, y_{\Lambda}^{i}\right)$, where $\Lambda=\left(\lambda_{k} \ldots \lambda_{1}\right), k=1, \ldots, r$, is a symmetric multi-index. We use the notation $\lambda+\Lambda=$ $\left(\lambda \lambda_{k} \ldots \lambda_{1}\right)$ and

$$
d_{\lambda}=\partial_{\lambda}+\sum_{0 \leq|\Lambda|} y_{\lambda+\Lambda}^{i} \partial_{i}^{\Lambda}, \quad d_{\Lambda}=d_{\lambda_{r}} \circ \cdots \circ d_{\lambda_{1}}
$$

A Lagrangian system on a fiber bundle is said to be a gauge system if its Lagrangian $L$ admits a gauge symmetry depending on parameter functions and their derivatives. In order to describe a gauge system, let us consider Lagrangian formalism on the bundle product

$$
E=Y \underset{X}{\times} W
$$


where $W \rightarrow X$ is a vector bundle whose sections are gauge parameter functions [8]. Let $W \rightarrow X$ be coordinated by $\left(x^{\lambda}, \xi^{r}\right)$. Then a gauge symmetry is represented by a differential operator

$$
v=\sum_{0 \leq|\Lambda| \leq m} v_{r}^{i, \Lambda}\left(x^{\lambda}, y_{\Sigma}^{i}\right) \xi_{\Lambda}^{r} \partial_{i}
$$

on $E$ (3) which is linear on $W$ and takes its values in the vertical tangent bundle $V Y$ of $Y \rightarrow X$. By means of a replacement of even gauge parameters $\xi^{r}$ and their jets $\xi_{\Lambda}^{r}$ with the odd ghosts $c^{r}$ and their jets $c_{\Lambda}^{r}$, the operator (4) defines a graded derivation

$$
v=\sum_{0 \leq|\Lambda| \leq m} v_{r}^{i, \Lambda}\left(x^{\lambda}, y_{\Sigma}^{i}\right) c_{\Lambda}^{r} \partial_{i}
$$

of the algebra of the original even fields and odd ghosts. Its extension

$$
v=\sum_{0 \leq|\Lambda| \leq m} v_{r}^{i, \Lambda} c_{\Lambda}^{r} \partial_{i}+u^{r} \partial_{r}
$$

to ghosts is called the BRST transformation if it is nilpotent. Such an extension exists if the original gauge transformations form an algebra [9].

As was mentioned above, gauge transformations in gauge gravitation theory are parameterized by vector fields on $X$. In this case, the vector bundle $W \rightarrow X$ possesses the composite fibration $W \rightarrow T X \rightarrow X$, where $T X$ is the tangent bundle of $X$. Here, we are concerned with the following three gauge gravitation theories: (i) the metric-affine gravitation theory on natural bundles describing gravity in the absence of matter fields, (ii) gauge theory on principal bundles with non-vertical gauge transformations which describes gauge potentials of internal symmetries in the presence of a gravitational field, (iii) gauge gravitation theory in the presence of Dirac fermion fields. The corresponding gauge transformations (12), (16), (38), (39), gauge symmetries (59), (61), (63), (65), and BRST symmetries (87), (88), (89) are obtained. However, we apply the BV quantization procedure only to the first of these gauge models describing pure gravity. We construct its extended Lagrangian (98), depending on ghost and antifields, and then the gauge-fixed Lagrangian (104), where antifields are replaced with gauge-fixing terms.

\section{Gauge gravitation theories}

Classical theory of gravity in the absence of matter fields can be formulated as a gauge theory on natural bundles over an oriented four-dimensional manifold $X$. The group of automorphisms of a natural bundle contains the subgroup of general covariant transformations defined as the canonical lift of diffeomorphisms of $X$. They are gauge transformations of metric-affine gravitation theory on natural bundles whose variables are linear connections and pseudo-Riemannian metrics on $X$. 
Linear connections on $X$ (henceforth world connection) are connections on the principal bundle $L X$ of linear frames in the tangent bundle $T X$ of $X$ with the structure group $G L_{4}=G L^{+}(4, \mathbb{R})$. These connections are represented by sections of the quotient bundle

$$
C_{K}=J^{1} L X / G L_{4},
$$

where $J^{1} L X$ is the first order jet manifold of sections of $L X \rightarrow X[6,7]$. This fiber bundle is provided with bundle coordinates $\left(x^{\lambda}, k_{\lambda^{\nu}}{ }\right)$ such that, for any section $K$ of $C_{K} \rightarrow X$, its

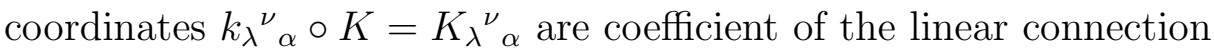

$$
K=d x^{\lambda} \otimes\left(\partial_{\lambda}+K_{\lambda}{ }^{\mu}{ }_{\nu} \dot{x}^{\nu} \dot{\partial}_{\mu}\right)
$$

on $T X$ with respect to the holonomic bundle coordinates $\left(x^{\lambda}, \dot{x}^{\lambda}\right)$. Note that the first order jet manifold $J^{1} C_{K}$ of the fiber bundle (7) possesses the canonical decomposition taking the coordinate form

$$
\begin{aligned}
& k_{\lambda \mu}{ }^{\alpha}{ }_{\beta}=\frac{1}{2}\left(R_{\lambda \mu}{ }^{\alpha}{ }_{\beta}+S_{\lambda \mu}{ }^{\alpha}{ }^{\beta}\right), \\
& R_{\lambda \mu}{ }^{\alpha}{ }_{\beta}=k_{\lambda \mu}{ }^{\alpha}{ }_{\beta}-k_{\mu \lambda}{ }^{\alpha}{ }_{\beta}+k_{\mu}{ }^{\alpha}{ }_{\varepsilon} k_{\lambda \beta}{ }^{\varepsilon}-k_{\lambda}{ }^{\alpha}{ }_{\varepsilon} k_{\mu \beta}{ }^{\varepsilon} \text {, } \\
& S_{\lambda \mu}{ }^{\alpha}{ }_{\beta}=k_{\lambda \mu}{ }^{\alpha}{ }_{\beta}+k_{\mu \lambda}{ }^{\alpha}{ }_{\beta}-k_{\mu}{ }^{\alpha}{ }_{\varepsilon} k_{\lambda}{ }^{\varepsilon} \beta+k_{\lambda}{ }^{\alpha}{ }_{\varepsilon} k_{\mu \beta}{ }^{\varepsilon} \text {. }
\end{aligned}
$$

If $K$ is a section of $C_{K} \rightarrow X$, then $R \circ K$ is the curvature of the world connection $K$.

In order to describe gravity, we assume that the linear frame bundle $L X$ admits a Lorentz structure, i.e., a principal subbundle $L^{h} X \subset L X$ whose structure group is the proper Lorentz group $\mathrm{L}=S O^{0}(1,3)$. By virtue of the well-known theorem [10], there is one-to-one correspondence between these subbundles and the global sections $h$ of the quotient bundle

$$
\Sigma_{T}=L X / \mathrm{L} .
$$

Namely, $L^{h} X=h^{*} L X$ is the bull-back of $L X \rightarrow \Sigma_{T}$ by $h$. We agree to call $\Sigma_{T}$ the tetrad bundle. This is the twofold of the metric bundle

$$
\Sigma=L X / S O(1,3),
$$

whose sections are pseudo-Riemannian (henceforth world) metrics on $X$. Being an open subbundle of the tensor bundle $\stackrel{2}{V}^{2} X$, the bundle $\Sigma(11)$ is provided with bundle coordinates $\left(x^{\lambda}, \sigma^{\mu \nu}\right)$.

The configuration space of gauge gravitation theory in the absence of matter fields is the bundle product $\Sigma \times C_{K}$. This is a natural bundle admitting the canonical lift

$$
\begin{aligned}
u_{K \Sigma} & =u^{\mu} \partial_{\mu}+\left(\sigma^{\nu \beta} \partial_{\nu} u^{\alpha}+\sigma^{\alpha \nu} \partial_{\nu} u^{\beta}\right) \frac{\partial}{\partial \sigma^{\alpha \beta}}+ \\
& \left(\partial_{\nu} u^{\alpha} k_{\mu}{ }^{\nu}{ }_{\beta}-\partial_{\beta} u^{\nu} k_{\mu}{ }^{\alpha}{ }_{\nu}-\partial_{\mu} u^{\nu} k_{\nu}{ }^{\alpha}{ }_{\beta}+\partial_{\mu \beta} u^{\alpha}\right) \frac{\partial}{\partial k_{\mu}{ }^{\alpha} \beta}
\end{aligned}
$$


of vector fields $u=u^{\lambda} \partial_{\lambda}$ on $X$ [6]. This lift is a Lie algebra homomorphism, and the vector fields (12) are infinitesimal generators of local one-dimensional groups of general covariant transformations, whose gauge parameters are vector fields on $X$.

Dealing with non-vertical automorphisms of non-natural bundles, one also comes to gravitation theory. For instance, let us consider the gauge theory on a principal bundle $P \rightarrow X$ with a structure Lie group $G$. In a general setting, the gauge-natural prolongations of $P$ and the associated natural-gauge bundles are called into play [11, 12]. Principal connections on $P$ are represented by sections of the quotient

$$
C=J^{1} P / G \rightarrow X
$$

called the bundle of principal connections [6]. This is an affine bundle coordinated by $\left(x^{\lambda}, a_{\lambda}^{r}\right)$ such that, given a section $A$ of $C \rightarrow X$, its components $A_{\lambda}^{r}=a_{\lambda}^{r} \circ A$ are coefficients of the familiar local connection form (i.e., gauge potentials). Infinitesimal generators of local one-parameter groups of automorphisms of a principal bundle $P$ are $G$-invariant projectable vector fields on $P \rightarrow X$. They are represented by sections of the vector bundle $T_{G} P=$ $T P / G \rightarrow X$. This fiber bundle is endowed with the coordinates $\left(x^{\lambda}, \tau^{\lambda}=\dot{x}^{\lambda}, \xi^{r}\right)$ with respect to the fiber bases $\left\{\partial_{\lambda}, e_{r}\right\}$ for $T_{G} P$, where $\left\{e_{r}\right\}$ is the basis for the right Lie algebra $\mathfrak{g}$ of $G$ such that $\left[e_{p}, e_{q}\right]=c_{p q}^{r} e_{r}$. The bracket of sections of $T_{G} P \rightarrow X$ reads

$$
\begin{aligned}
& u_{P}=u^{\lambda} \partial_{\lambda}+u^{r} e_{r}, \quad v_{P}=v^{\lambda} \partial_{\lambda}+v^{r} e_{r}, \\
& {\left[u_{P}, v_{P}\right]=\left(u^{\mu} \partial_{\mu} v^{\lambda}-v^{\mu} \partial_{\mu} u^{\lambda}\right) \partial_{\lambda}+\left(u^{\lambda} \partial_{\lambda} v^{r}-v^{\lambda} \partial_{\lambda} u^{r}+c_{p q}^{r} u^{p} v^{q}\right) e_{r} .}
\end{aligned}
$$

Any section $u_{P}$ of the vector bundle $T_{G} P \rightarrow X$ yields the vector field

$$
u_{C}=u^{\lambda} \partial_{\lambda}+\left(c_{p q}^{r} a_{\lambda}^{p} u^{q}+\partial_{\lambda} u^{r}-a_{\mu}^{r} \partial_{\lambda} u^{\mu}\right) \partial_{r}^{\lambda}
$$

on the bundle of principal connections $C$ (13). For instance, this vector field is a gauge symmetry of the global Chern-Simons Lagrangian in gauge theory on a principal bundle with a structure semi-simple Lie group $G$ over a three-dimensional base $X$ [13]. In order to obtain a gauge symmetry of the Yang-Mills Lagrangian, one have to extend the vector field (15) to the vector field

$$
u_{C \Sigma}=u^{\lambda} \partial_{\lambda}+\left(c_{p q}^{r} a_{\lambda}^{p} u^{q}+\partial_{\lambda} u^{r}-a_{\mu}^{r} \partial_{\lambda} u^{\mu}\right) \partial_{r}^{\lambda}+\left(\sigma^{\nu \beta} \partial_{\nu} u^{\alpha}+\sigma^{\alpha \nu} \partial_{\nu} u^{\beta}\right) \frac{\partial}{\partial \sigma^{\alpha \beta}}
$$

on the bundle product $C \times \Sigma$ of $C$ and the metric bundle $\Sigma(11)$.

The physical underlying reasons for the existence of a Lorentz structure and, consequently, a world metric are Dirac fermion fields. They are described by sections of Dirac spinor bundles, which fail to be natural. In order to develop gauge theory of gravity in the presence of Dirac fermion fields, one has to define general covariant transformations of these fields. 
Recall that Dirac spinors are described in terms of Clifford algebras as follows $[14,15]$. Let $M$ be the Minkowski space equipped with the Minkowski metric $\eta$ of signature (+---), and let $\left\{t^{a}\right\}$ be a fixed basis for $M$. The complex Clifford algebra $\mathbb{C}_{1,3}$ is defined as the complexified quotient of the tensor algebra $\otimes M$ of $M$ by the two-sided ideal generated by elements

$$
t \otimes t^{\prime}+t^{\prime} \otimes t-2 \eta\left(t, t^{\prime}\right) \in \otimes M, \quad t, t^{\prime} \in M .
$$

The Dirac spinor space $V_{s}$ is introduced as a minimal left ideal of $\mathbb{C}_{1,3}$ on which this algebra acts by left multiplications. There is the representation

$$
\gamma: M \otimes V \rightarrow V, \quad \gamma\left(t^{a}\right)=\gamma^{a},
$$

of elements of the Minkowski space $M \subset \mathbb{C}_{1,3}$ by the Dirac $\gamma$-matrices on $V_{s}$. Different ideals $V_{s}$ lead to equivalent representations (17). The complex Clifford algebra $\mathbb{C}_{1,3}$ is isomorphic to the real Clifford algebra $\mathbb{R}_{2,3}$, whose generating space is $\mathbb{R}^{5}$ endowed with the metric of signature $(+---+)$. Its subalgebra generated by elements of $M \subset \mathbb{R}^{5}$ is the real Clifford algebra $\mathbb{R}_{1,3}$. The Clifford group $G_{1,3}$ consists of the invertible elements $l_{s}$ of $\mathbb{R}_{1,3}$ such that the inner automorphisms defined by these elements preserve the Minkowski space $M \subset \mathbb{R}_{1,3}$. Since this action of $G_{1,3}$ on $M$ is not effective, one consider its spin subgroup $\mathrm{L}_{s}=S L(2, \mathbb{C})$, acting on the spinor space $V_{s}$ by means of the generators

$$
L_{a b}=\frac{1}{4}\left[\gamma_{a}, \gamma_{b}\right]
$$

This action preserves the representation (17) and the spinor metric

$$
a\left(v, v^{\prime}\right)=\frac{1}{2}\left(v^{+} \gamma^{0} v^{\prime}+v^{\prime+} \gamma^{0} v\right), \quad v, v^{\prime} \in V_{s} .
$$

The spin group $\mathrm{L}_{s}$ is the universal twofold of the proper Lorentz group L. Given the universal twofold $\widetilde{G L}$ of the group $G L_{4}$, we have the commutative diagram

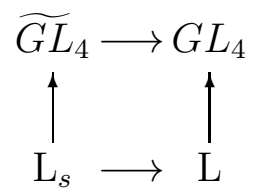

Note that the group $\widetilde{G L}_{4}$ admits spinor representations, but they are infinite-dimensional $[2,16]$. Let us consider the $\widetilde{G L}$-principal bundle $\widetilde{L X} \rightarrow X$ which is the twofold of the linear frame bundle $L X[17,18,19]$. We agree to call it the spin frame bundle. One says that $\widetilde{L X}$ admits a Dirac spin structure if there exists its $\mathrm{L}_{s}$-principal subbundle $P^{h}$. Then we have the commutative diagram

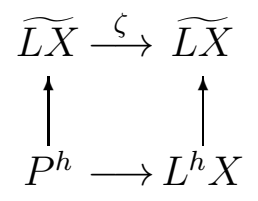


where $L^{h} X$ is some Lorentz structure $[20,21]$. As a consequence, $\widetilde{L X} / \mathrm{L}_{s}=\Sigma_{T}(10)$, and there is one-to-one correspondence $P^{h}=h^{*} \widetilde{L X}$ between the Dirac spin structures $P^{h} \subset \widetilde{L X}$ and the global sections $h$ of the tetrad bundle $\Sigma_{T} \rightarrow X$. We agree to call these sections the tetrad fields because of the following.

With respect to the holonomic frames $\left\{\partial_{\mu}\right\}$ in the tangent bundle $T X$, every element $\left\{H_{a}\right\}$ of the linear frame bundle $L X$ takes the form $H_{a}=H_{a}^{\mu} \partial_{\mu}$. The matrix elements $H_{a}^{\mu}$ constitute the bundle coordinates on $L X \rightarrow X$ such that the transition functions and the canonical right action of $G L_{4}$ on $L X$ take the form

$$
H_{a}^{\prime \mu}=\frac{\partial x^{\prime \mu}}{\partial x^{\lambda}} H_{a}^{\lambda}, \quad G L_{4} \ni g: H_{a}^{\mu} \mapsto H_{b}^{\mu} g_{a}^{b} .
$$

Let collections of local sections $\widetilde{\Psi}=\left\{z_{\epsilon}^{s}\right\}$ and $\Psi=\left\{z_{\epsilon}=\zeta \circ z_{\epsilon}^{s}\right\}$ yield associated atlases of the principal bundles $\widetilde{L X} \rightarrow \Sigma_{T}$ and $L X \rightarrow \Sigma_{T}$, respectively. Then the tetrad bundle $\Sigma_{T} \rightarrow X$ can be provided with bundle coordinates $\left(x^{\lambda}, \sigma_{a}^{\mu}=H_{a}^{\mu} \circ \zeta_{e}\right)$. Components of a tetrad field $h$ with respect to these coordinates are tetrad functions $h_{a}^{\mu}=\sigma_{\mu}^{a} \circ h$. The familiar relation $g^{\mu \nu}=h_{a}^{\mu} h_{b}^{\nu} \eta_{a b}$ between tetrad functions and metric functions of the world metric $g: X \stackrel{h}{\longrightarrow} \Sigma_{T} \rightarrow \Sigma$ holds.

There is the well-known topological obstruction to the existence of a Dirac spin structure. Let us restrict our consideration to non-compact manifolds $X$. Then a Dirac spinor structure exists iff $X$ is parallelizable [22] (e.g., $X=\mathbb{R} \times Z$ because any orientable threedimensional manifold $Z$ is parallelizable [23]). In this case, the spin frame bundle is unique and all Dirac spin structures are equivalent $[22,24]$, i.e., all $\mathrm{L}_{s}$-principal subbundles $P^{h}$ of $\widetilde{L X}$ are pairwise isomorphic by means of automorphisms of $\widetilde{L X}$ and isomorphic to the trivial $\mathrm{L}_{s}$-principal bundle $P^{0} \rightarrow X$.

Since $\widetilde{L X} \rightarrow \Sigma_{T}$ is an $\mathrm{L}_{s}$-principal bundle, one can consider the associated spinor bundle

$$
S=\left(\widetilde{L X} \times V_{s}\right) / \mathrm{L}_{s} \rightarrow \Sigma_{T}
$$

over $\Sigma_{T}[6,21]$. We agree to call it the universal spinor bundle because, given a tetrad field $h$, the pull-back $S^{h}=h^{*} S \rightarrow X$ of $S$ onto $X$ is a spinor bundle on $X$ which is associated to the $\mathrm{L}_{s}$-principal bundle $P^{h}$. Sections of $S^{h}$ describe Dirac fermion fields in the presence of a tetrad gravitational field $h$. Given an atlas $\widetilde{\Psi}$ of $\widetilde{L X}$ and the corresponding bundle coordinates $\left(x^{\lambda}, \sigma_{a}^{\mu}\right)$ on $\Sigma_{T}$, the universal spinor bundle $S(22)$ is endowed with bundle coordinates $\left(x^{\lambda}, \sigma_{a}^{\mu}, y^{A}\right)$, where $y^{A}$ are coordinates on the spinor space $V_{s}$.

The universal spinor bundle $S \rightarrow \Sigma_{T}$ is a subbundle of the bundle of Clifford algebras which is generated by the bundle of Minkowski spaces

$$
E_{M}=(L X \times M) / \mathrm{L} \rightarrow \Sigma_{T}
$$


associated with the L-principal bundle $L X \rightarrow \Sigma_{T}$. Then due to the representation $\gamma(17)$, there exists the representation

$$
\gamma_{\Sigma}: T^{*} X \underset{\Sigma_{T}}{\otimes} S \longrightarrow(\widetilde{L X} \times(M \otimes V)) / \mathrm{L}_{s} \rightarrow(\widetilde{L X} \times \gamma(M \otimes V)) / \mathrm{L}_{s}=S
$$

defined by the coordinate expression $\gamma_{\Sigma}\left(d x^{\lambda}\right)=\sigma_{a}^{\lambda} \gamma^{a}$.

Given a world connection $K$ on $X$, the universal spinor bundle $S \rightarrow \Sigma_{T}$ is provided with the connection

$$
\begin{gathered}
A_{\Sigma}=d x^{\lambda} \otimes\left(\partial_{\lambda}-\frac{1}{4}\left(\eta^{k b} \sigma_{\mu}^{a}-\eta^{k a} \sigma_{\mu}^{b}\right) \sigma_{k}^{\nu} K_{\lambda}{ }^{\mu}{ }_{\nu} L_{a b}{ }_{B}{ }_{B} y^{B} \partial_{A}\right)+ \\
d \sigma_{k}^{\mu} \otimes\left(\partial_{\mu}^{k}+\frac{1}{4}\left(\eta^{k b} \sigma_{\mu}^{a}-\eta^{k a} \sigma_{\mu}^{b}\right) L_{a b}{ }_{B}{ }_{B} y^{B} \partial_{A}\right) .
\end{gathered}
$$

Given a tetrad field $h$, its restriction to $S^{h}$ is the familiar spin connection

$$
K_{h}=d x^{\lambda} \otimes\left[\partial_{\lambda}+\frac{1}{4}\left(\eta^{k b} h_{\mu}^{a}-\eta^{k a} h_{\mu}^{b}\right)\left(\partial_{\lambda} h_{k}^{\mu}-h_{k}^{\nu} K_{\lambda}{ }^{\mu}{ }_{\nu}\right) L_{a b}{ }^{A}{ }_{B} y^{B} \partial_{A}\right]
$$

defined by a world connection $K[25,26]$. The connection (25) yields the first order differential operator

$$
\begin{aligned}
& D:: J^{1} S \rightarrow T^{*} X \underset{\Sigma_{T}}{\otimes} S, \\
& D=d x^{\lambda} \otimes\left[y_{\lambda}^{A}-\frac{1}{4}\left(\eta^{k b} \sigma_{\mu}^{a}-\eta^{k a} \sigma_{\mu}^{b}\right)\left(\sigma_{\lambda k}^{\mu}-\sigma_{k}^{\nu} K_{\lambda}{ }^{\mu}{ }_{\nu}\right) L_{a b}{ }^{A}{ }_{B} y^{B}\right] \partial_{A},
\end{aligned}
$$

on the fiber bundle $S \rightarrow X$. Its restriction to $J^{1} S^{h} \subset J^{1} S$ recovers the familiar covariant differential on the spinor bundle $S^{h} \rightarrow X$ relative to the spin connection (26). Combining (24) and (27) gives the first order differential operator

$$
\begin{aligned}
& \mathcal{D}=\gamma_{\Sigma} \circ D: J^{1} S \rightarrow T^{*} X \underset{\Sigma_{T}}{\otimes} S \rightarrow S, \\
& \mathcal{D}=\sigma_{a}^{\lambda} \gamma^{a B}{ }_{A}\left[y_{\lambda}^{A}-\frac{1}{4}\left(\eta^{k b} \sigma_{\mu}^{a}-\eta^{k a} \sigma_{\mu}^{b}\right)\left(\sigma_{\lambda k}^{\mu}-\sigma_{k}^{\nu} K_{\lambda}{ }^{\mu}{ }_{\nu}\right) L_{a b}{ }^{A}{ }_{B} y^{B}\right],
\end{aligned}
$$

on the fiber bundle $S \rightarrow X$. Its restriction to $J^{1} S^{h} \subset J^{1} S$ is the familiar Dirac operator on the spinor bundle $S^{h}$ in the presence of a background tetrad field $h$ and a world connection $K$.

Thus, we come to the model of the metric-affine gravity and Dirac fermion fields. Its configuration space is the bundle product

$$
Y=C_{K} \underset{\Sigma_{T}}{\times} S
$$


coordinated by $\left(x^{\mu}, \sigma_{a}^{\mu}, k_{\mu}{ }^{\alpha}, y^{A}\right)$. Let $J_{\Sigma_{T}}^{1} Y$ denote the first order jet manifold of the fiber bundle $Y \rightarrow \Sigma_{T}$. This fiber bundle can be endowed with the spin connection

$$
\begin{gathered}
A_{Y}=d x^{\lambda} \otimes\left(\partial_{\lambda}-\frac{1}{4}\left(\eta^{k b} \sigma_{\mu}^{a}-\eta^{k a} \sigma_{\mu}^{b}\right) \sigma_{k}^{\nu} k_{\lambda}{ }^{\mu}{ }_{\nu} L_{a b}{ }^{A}{ }_{B} y^{B} \partial_{A}\right)+ \\
d \sigma_{k}^{\mu} \otimes\left(\partial_{\mu}^{k}+\frac{1}{4}\left(\eta^{k b} \sigma_{\mu}^{a}-\eta^{k a} \sigma_{\mu}^{b}\right) L_{a b}{ }_{B}{ }_{B} y^{B} \partial_{A}\right) .
\end{gathered}
$$

With this connection, we obtain the first order differential operator

$$
D_{Y}=d x^{\lambda} \otimes\left[y_{\lambda}^{A}-\frac{1}{4}\left(\eta^{k b} \sigma_{\mu}^{a}-\eta^{k a} \sigma_{\mu}^{b}\right)\left(\sigma_{\lambda k}^{\mu}-\sigma_{k}^{\nu} k_{\lambda}{ }^{\mu}{ }_{\nu}\right) L_{a b}{ }^{A}{ }_{B} y^{B}\right] \partial_{A},
$$

and the total Dirac operator

$$
\mathcal{D}_{Y}=\sigma_{a}^{\lambda} \gamma^{a B}{ }_{A}\left[y_{\lambda}^{A}-\frac{1}{4}\left(\eta^{k b} \sigma_{\mu}^{a}-\eta^{k a} \sigma_{\mu}^{b}\right)\left(\sigma_{\lambda k}^{\mu}-\sigma_{k}^{\nu} k_{\lambda}{ }^{\mu}{ }_{\nu}\right) L_{a b}{ }^{A}{ }_{B} y^{B}\right]
$$

on the fiber bundle $Y \rightarrow X$. Given a world connection $K: X \rightarrow C_{K}$, the restrictions of the spin connection $A_{Y}(30)$, the operators $D_{Y}(31)$ and $\mathcal{D}_{Y}(32)$ to $K^{*} Y$ are exactly the spin connection (25), the operators (27) and (28), respectively. The total Lagrangian of the metric-affine gravity and fermion fields is the sum of a metric-affine Lagrangian $L_{\mathrm{MA}}$ depending on the variables $\sigma^{\mu \nu}=\sigma_{a}^{\mu} \sigma_{b}^{\nu} \eta^{a b}, k_{\mu}{ }^{\alpha}$ and the Dirac Lagrangian constructed from the Dirac operator (32) and the spinor metric (19) [6, 21].

Turn now to gauge transformations in this gauge theory. The spin frame bundle $\widetilde{L X} \rightarrow$ $X$ as like as the linear frame one $L X \rightarrow X$ is natural, and it admits the canonical lift of any vector field on $X$. Hence, the universal spinor bundle $S \rightarrow X$ is also natural, and the canonical lift $u_{S}$ onto $S$ of vector fields on $X$ exists. The goal is to find its coordinate expression. Difficulties arise because the tetrad coordinates $\sigma_{a}^{\mu}$ on $\Sigma_{T}$ depend on the choice of an atlas $\Psi$ of the bundle $L X$. Therefore, non-canonical components appear in the coordinate expression of $u_{S}[21,26]$.

Since the fiber bundles $L X \rightarrow X, G L_{4} \rightarrow G L_{4} / \mathrm{L}$ and, accordingly, the fiber bundles $\widetilde{L X} \rightarrow X, \widetilde{G L}_{4} \rightarrow \mathrm{L}_{s}$ are trivial, the fiber bundles $\widetilde{L X} \rightarrow \Sigma_{T}$ and $S \rightarrow \Sigma_{T}$ are also trivial. Let $\Psi$ be an atlas of $\widetilde{L X} \rightarrow \Sigma_{T}$ with transition functions constant on fibers of $\Sigma_{T}$. This atlas provides a trivialization $\widetilde{L X} \cong P^{0} \times \Sigma_{T}$ and the corresponding trivialization

$$
S \cong S^{0} \times \Sigma_{T}
$$

of the universal spinor bundle. With respect to such an atlas, one can define the lift

$$
\begin{aligned}
u_{S}= & u^{\lambda} \partial_{\lambda}+\partial_{\nu} u^{\alpha} \sigma_{c}^{\nu} \partial_{\alpha}^{c}+ \\
& \frac{1}{4}\left(\eta^{k b} \sigma_{\mu}^{a}-\eta^{k a} \sigma_{\mu}^{b}\right)\left(\partial_{\nu} u^{\mu} \sigma_{k}^{\nu}-u^{\nu} \sigma_{\nu k}^{\mu}\right)\left(-L_{a b}{ }_{c}^{d} \sigma_{d}^{\alpha} \partial_{\alpha}^{c}+L_{a b}{ }^{A}{ }_{B} y^{B} \partial_{A}\right), \\
& L_{a b}{ }^{d}{ }_{c}=\eta_{b c} \delta_{a}^{d}-\eta_{a c} \delta_{b}^{d}
\end{aligned}
$$


onto $S$ of vector fields $u=u^{\lambda} \partial_{\lambda}$ on $X$. This lift however is not a vector field because it depends on the jet coordinates $\sigma_{\nu k}^{\mu}$. It is a generalized vector field (see the next Section). Its projection onto $S^{h}$ in accordance with the splitting (33) is the well-known Lie derivative

$$
u_{h}=u^{\lambda} \partial_{\lambda}+\frac{1}{4}\left(\eta^{k b} h_{\mu}^{a}-\eta^{k a} h_{\mu}^{b}\right)\left(\partial_{\nu} u^{\mu} h_{k}^{\nu}-u^{\nu} \partial_{\nu} h_{k}^{\mu}\right) L_{a b}{ }_{B}{ }_{B} y^{B} \partial_{A}
$$

of spinor fields in the presence of a tetrad field $h[27,28]$. Note that the lift (34) fails to be a Lie algebra homomorphism.

At the same time, one can think of the fiber bundle $S \rightarrow X(33)$ as being associated with the $\mathrm{L}_{s}$-principal bundle $P^{0} \rightarrow X$. Infinitesimal generators of local one-parameter groups of automorphisms of $P^{0}$ are represented by sections of the vector bundle $T P^{0} / \mathrm{L}_{s} \rightarrow X$ which take the coordinate form $u_{P}=u^{\lambda} \partial_{\lambda}+u^{m} e_{m}$, where $\left\{e_{m}\right\}$ is the basis for the Lie algebra of $\mathrm{L}_{s}$. Any vector field $u_{P}$ yields the vector field

$$
u_{S}=u^{\lambda} \partial_{\lambda}+\left(\partial_{\nu} u^{\alpha} \sigma_{c}^{\nu}-u^{m} I_{m}{ }^{d}{ }_{c} \sigma_{d}^{\alpha}\right) \partial_{\alpha}^{c}+u^{m} I_{m}{ }^{A}{ }_{B} y^{B} \partial_{A}
$$

on the associated bundle $S$. These vector fields can also be regarded as infinitesimal gauge transformations of gauge theory of gravity in the presence of Dirac fermion fields. The total configuration space of this theory is the bundle product

$$
Y=S \underset{\Sigma_{T}}{\times} C_{K}
$$

coordinated by $\left(x^{\lambda}, \sigma_{a}^{\mu}, k_{\mu}{ }^{\alpha}{ }_{\beta}, y^{A}\right)$. The generalized vector fields (34) and the vector fields (36) are extended to the fiber bundle (37) as

$$
\begin{aligned}
u_{S K}=u^{\lambda} \partial_{\lambda}+\partial_{\nu} u^{\alpha} \sigma_{c}^{\nu} \partial_{\alpha}^{c}+ & \\
& \frac{1}{4}\left(\eta^{k b} \sigma_{\mu}^{a}-\eta^{k a} \sigma_{\mu}^{b}\right)\left(\partial_{\nu} u^{\mu} \sigma_{k}^{\nu}-u^{\nu} \sigma_{\nu k}^{\mu}\right)\left(-L_{a b}{ }_{c}{ } \sigma_{d}^{\alpha} \partial_{\alpha}^{c}+L_{a b}{ }_{B}{ }_{B} y^{B} \partial_{A}\right)+ \\
& {\left[\partial_{\nu} u^{\alpha} k_{\mu}{ }^{\nu}{ }_{\beta}-\partial_{\beta} u^{\nu} k_{\mu}{ }^{\alpha}{ }_{\nu}-\partial_{\mu} u^{\nu} k_{\nu}{ }^{\alpha}{ }_{\beta}+\partial_{\mu \beta} u^{\alpha}\right] \frac{\partial}{\partial k_{\mu}{ }^{\alpha} \beta}, } \\
u_{S K}= & u^{\lambda} \partial_{\lambda}+\left(\partial_{\nu} u^{\alpha} \sigma_{c}^{\nu}-u^{m} I_{m}{ }^{d}{ }_{c} \sigma_{d}^{\alpha}\right) \partial_{\alpha}^{c}+u^{m} I_{m}{ }^{A}{ }_{B} y^{B} \partial_{A}+ \\
& {\left[\partial_{\nu} u^{\alpha} k_{\mu}{ }^{\nu}{ }_{\beta}-\partial_{\beta} u^{\nu} k_{\mu}{ }^{\alpha}{ }_{\nu}-\partial_{\mu} u^{\nu} k_{\nu}{ }^{\alpha}{ }_{\beta}+\partial_{\mu \beta} u^{\alpha}\right] \frac{\partial}{\partial k_{\mu}{ }^{\alpha}{ }_{\beta}} . }
\end{aligned}
$$

\section{Gauge symmetries}

In a general setting, gauge systems on fiber bundle $Y \rightarrow X$ are described as follows [8].

With the inverse system of jet manifolds

$$
X \stackrel{\pi}{\longleftarrow} Y \stackrel{\pi_{0}^{1}}{\longleftarrow} J^{1} Y \longleftarrow \cdots J^{r-1} Y \stackrel{\pi_{r-1}^{r}}{\longleftarrow} J^{r} Y \longleftarrow \cdots,
$$


one has the direct system

$$
\mathcal{O}^{*} X \stackrel{\pi^{*}}{\longrightarrow} \mathcal{O}^{*} Y \stackrel{\pi_{0}^{1 *}}{\longrightarrow} \mathcal{O}_{1}^{*} Y \longrightarrow \cdots \mathcal{O}_{r-1}^{*} Y \stackrel{\pi_{r-1}^{r}}{\longrightarrow} \mathcal{O}_{r}^{*} Y \longrightarrow \cdots
$$

of graded differential algebras (henceforth GDAs) $\mathcal{O}_{r}^{*} Y$ of exterior forms on jet manifolds $J^{r} Y$ with respect to the pull-back monomorphisms $\pi_{r-1}^{r}{ }^{*}$. Its direct limit $\mathcal{O}_{\infty}^{*} Y$ is a GDA consisting of all exterior forms on finite order jet manifolds modulo the pull-back identification.

The projective limit $\left(J^{\infty} Y, \pi_{r}^{\infty}: J^{\infty} Y \rightarrow J^{r} Y\right)$ of the inverse system (40) is a Fréchet manifold. A bundle atlas $\left\{\left(U_{Y} ; x^{\lambda}, y^{i}\right)\right\}$ of $Y \rightarrow X$ yields the coordinate atlas

$$
\left\{\left(\left(\pi_{0}^{\infty}\right)^{-1}\left(U_{Y}\right) ; x^{\lambda}, y_{\Lambda}^{i}\right)\right\}, \quad y_{\lambda+\Lambda}^{\prime i}=\frac{\partial x^{\mu}}{\partial x^{\prime \lambda}} d_{\mu} y_{\Lambda}^{i}, \quad 0 \leq|\Lambda|,
$$

of $J^{\infty} Y$, where $d_{\mu}$ are the total derivatives (2). Then $\mathcal{O}_{\infty}^{*} Y$ can be written in a coordinate form where the horizontal one-forms $\left\{d x^{\lambda}\right\}$ and the contact one-forms $\left\{\theta_{\Lambda}^{i}=d y_{\Lambda}^{i}-y_{\lambda+\Lambda}^{i} d x^{\lambda}\right\}$ are local generating elements of the $\mathcal{O}_{\infty}^{0} Y$-algebra $\mathcal{O}_{\infty}^{*} Y$. There is the canonical decomposition $\mathcal{O}_{\infty}^{*} Y=\oplus \mathcal{O}_{\infty}^{k, m} Y$ of $\mathcal{O}_{\infty}^{*} Y$ into $\mathcal{O}_{\infty}^{0} Y$-modules $\mathcal{O}_{\infty}^{k, m} Y$ of $k$-contact and $m$-horizontal forms together with the corresponding projectors $h_{k}: \mathcal{O}_{\infty}^{*} Y \rightarrow \mathcal{O}_{\infty}^{k, *} Y$. Accordingly, the exterior differential on $\mathcal{O}_{\infty}^{*} Y$ is split into the sum $d=d_{H}+d_{V}$ of the nilpotent total and vertical differentials

$$
d_{H}(\phi)=d x^{\lambda} \wedge d_{\lambda}(\phi), \quad d_{V}(\phi)=\theta_{\Lambda}^{i} \wedge \partial_{i}^{\Lambda} \phi, \quad \phi \in \mathcal{O}_{\infty}^{*} Y
$$

Any finite order Lagrangian (1) is an element of $\mathcal{O}_{\infty}^{0, n} Y$, while

$$
\delta L=\mathcal{E}_{i} \theta^{i} \wedge \omega=\sum_{0 \leq|\Lambda|}(-1)^{|\Lambda|} d_{\Lambda}\left(\partial_{i}^{\Lambda} \mathcal{L}\right) \theta^{i} \wedge \omega \in \mathcal{O}_{\infty}^{1, n} Y
$$

is its Euler-Lagrange operator taking values into the vector bundle

$$
V^{*} Y \underset{Y}{\otimes} \wedge T^{*} X
$$

where $V^{*} Y$ denotes the vertical cotangent bundle of $Y \rightarrow X$. Given a Lagrangian $L$ and its Euler-Lagrange operator $\delta L$ (43), we further abbreviate $A \approx 0$ with an equality which holds on-shell. This means that $A$ is an element of a module over the ideal $I_{L}$ of the ring $\mathcal{O}_{\infty}^{0} Y$ which is locally generated by the variational derivatives $\mathcal{E}_{i}$ and their total derivations $d_{\Lambda} \mathcal{E}_{i}$. We say that $I_{L}$ is a differential ideal because, if a local function $f$ belongs to $I_{L}$, then every total derivative $d_{\Lambda} f$ does as well.

A Lagrangian system on a fiber bundle $Y \rightarrow X$ is said to be a gauge theory if its Lagrangian $L$ admits a family of variational symmetries parameterized by elements of a vector bundle $W \rightarrow X$ and its jet manifolds as follows. 
Let $\mathfrak{d} \mathcal{O}_{\infty}^{0} Y$ be the $\mathcal{O}_{\infty}^{0} Y$-module of derivations of the $\mathbb{R}$-ring $\mathcal{O}_{\infty}^{0} Y$. Any $\vartheta \in \mathfrak{d} \mathcal{O}_{\infty}^{0} Y$ yields the graded derivation (the interior product) $\vartheta\rfloor \phi$ of the GDA $\mathcal{O}_{\infty}^{*} Y$ given by the relations

$$
\left.\vartheta\rfloor d f=\vartheta(f), \quad \vartheta\rfloor(\phi \wedge \sigma)=(\vartheta\rfloor \phi) \wedge \sigma+(-1)^{|\phi|} \phi \wedge(\vartheta\rfloor \sigma\right), \quad f \in \mathcal{O}_{\infty}^{0} Y, \quad \phi, \sigma \in \mathcal{O}_{\infty}^{*} Y,
$$

and its derivation (the Lie derivative)

$$
\left.\left.\mathbf{L}_{\vartheta} \phi=\vartheta\right\rfloor d \phi+d(\vartheta\rfloor \phi\right), \quad \mathbf{L}_{\vartheta}\left(\phi \wedge \phi^{\prime}\right)=\mathbf{L}_{\vartheta}(\phi) \wedge \phi^{\prime}+\phi \wedge \mathbf{L}_{\vartheta}\left(\phi^{\prime}\right), \quad \phi, \phi^{\prime} \in \mathcal{O}_{\infty}^{*} Y .
$$

Relative to an atlas (42), a derivation $\vartheta \in \mathfrak{d} \mathcal{O}_{\infty}^{0}$ reads

$$
\vartheta=\vartheta^{\lambda} \partial_{\lambda}+\vartheta^{i} \partial_{i}+\sum_{|\Lambda|>0} \vartheta_{\Lambda}^{i} \partial_{i}^{\Lambda}
$$

where the tuple of derivations $\left\{\partial_{\lambda}, \partial_{i}^{\Lambda}\right\}$ is defined as the dual of that of the exterior forms $\left\{d x^{\lambda}, d y_{\Lambda}^{i}\right\}$ with respect to the interior product $\rfloor[29]$.

A derivation $\vartheta$ is called contact if the Lie derivative $\mathbf{L}_{\vartheta}(45)$ preserves the contact ideal of the GDA $\mathcal{O}_{\infty}^{*} Y$ generated by contact forms. A derivation $v(46)$ is contact iff

$$
\vartheta_{\Lambda}^{i}=d_{\Lambda}\left(\vartheta^{i}-y_{\mu}^{i} \vartheta^{\mu}\right)+y_{\mu+\Lambda}^{i} \vartheta^{\mu}, \quad 0<|\Lambda| .
$$

Any contact derivation admits the horizontal splitting

$$
\vartheta=\vartheta_{H}+\vartheta_{V}=\vartheta^{\lambda} d_{\lambda}+\left(v^{i} \partial_{i}+\sum_{0<|\Lambda|} d_{\Lambda} v^{i} \partial_{i}^{\Lambda}\right), \quad v^{i}=\vartheta^{i}-y_{\mu}^{i} \vartheta^{\mu} .
$$

Its vertical part $\vartheta_{V}$ is completely determined by the first summand

$$
v=v^{i}\left(x^{\lambda}, y_{\Lambda}^{i}\right) \partial_{i}, \quad 0 \leq|\Lambda| \leq k .
$$

This is a section of the pull-back $V Y \underset{Y}{\times} J^{k} Y \rightarrow J^{k} Y$, i.e., a $k$-order $V Y$-valued differential operator on $Y$. One calls $v(49)$ a generalized vector field on $Y$.

One can show that the Lie derivative of a Lagrangian $L(1)$ along a contact derivation $\vartheta$ (48) fulfills the first variational formula

$$
\left.\left.\left.\mathbf{L}_{\vartheta} L=v\right\rfloor \delta L+d_{H}\left(h_{0}(\vartheta\rfloor \Xi_{L}\right)\right)+\mathcal{L} d_{V}\left(\vartheta_{H}\right\rfloor \omega\right),
$$

where $\Xi_{L}$ is a Lepagean equivalent of $L$ [29]. A contact derivation $\vartheta(48)$ is called variational if the Lie derivative (50) is $d_{H}$-exact, i.e., $\mathbf{L}_{\vartheta} L=d_{H} \sigma, \sigma \in \mathcal{O}_{\infty}^{0, n-1}$. A glance at the expression (50) shows that: (i) $\vartheta$ (48) is variational only if it is projected onto $X$; (ii) $\vartheta$ is variational iff its vertical part $\vartheta_{V}$ is well; (iii) it is variational iff $\left.v\right\rfloor \delta L$ is $d_{H}$-exact. Therefore, we can restrict our consideration to vertical contact derivations $\vartheta=\vartheta_{V}$. A 
generalized vector field $v(49)$ is called a variational symmetry of a Lagrangian $L$ if it generates a variational contact derivation.

In order to define a gauge symmetry [8], let us consider the bundle product $E$ (3) coordinated by $\left(x^{\lambda}, y^{i}, \xi^{r}\right)$. Given a Lagrangian $L$ on $Y$, let us consider its pull-back, say again $L$, onto $E$. Let $\vartheta_{E}$ be a contact derivation of the $\mathbb{R}$-ring $\mathcal{O}_{\infty}^{0} E$, whose restriction

$$
\vartheta=\left.\vartheta_{E}\right|_{\mathcal{O}_{\infty}^{0} Y}=\sum_{0 \leq|\Lambda|} d_{\Lambda} v^{i} \partial_{i}^{\Lambda}
$$

to $\mathcal{O}_{\infty}^{0} Y \subset \mathcal{O}_{\infty}^{0} E$ is linear in coordinates $\xi_{\Xi}^{r}$. It is determined by a generalized vector field $v_{E}$ on $E$ whose projection

$$
v: J^{k} E \stackrel{v_{E}}{\longrightarrow} V E \rightarrow \underset{Y}{\times} \underset{Y}{\times} V
$$

is a linear $V Y$-valued differential operator $v(4)$ on $E$. Let $\vartheta_{E}$ be a variational symmetry of a Lagrangian $L$ on $E$, i.e.,

$$
\left.\left.v_{E}\right\rfloor \delta L=v\right\rfloor \delta L=d_{H} \sigma .
$$

Then one says that $v(4)$ is a gauge symmetry of a Lagrangian $L$.

In accordance with Noether's second theorem [8], if a Lagrangian $L$ (1) admits a gauge symmetry $v(4)$, its Euler-Lagrange operator (43) obeys the Noether identity

$$
\begin{aligned}
& \Delta \circ \delta L=0 \\
& {\left[\sum_{0 \leq|\Lambda| \leq m} \Delta_{r}^{i, \Lambda} d_{\Lambda} \mathcal{E}_{i}\right] \xi^{r} \omega=\left[\sum_{0 \leq|\Lambda| \leq m}(-1)^{|\Lambda|} d_{\Lambda}\left(v_{r}^{i, \Lambda} \mathcal{E}_{i}\right)\right] \xi^{r} \omega=0}
\end{aligned}
$$

where

$$
\begin{aligned}
& \Delta=\xi^{r} \Delta_{r} \omega=\sum_{0 \leq|\Lambda| \leq m} \xi^{r} \Delta_{r}^{i, \Lambda}\left(x^{\lambda}, y_{\Sigma}^{j}\right) \bar{y}_{\Lambda i} \omega=\xi^{r}\left[\sum_{0 \leq|\Lambda| \leq m}(-1)^{|\Lambda|} d_{\Lambda}\left(v_{r}^{i, \Lambda} \bar{y}_{i}\right)\right] \omega \\
& \Delta_{r}^{i, \Lambda}=\sum_{0 \leq|\Sigma| \leq m-|\Lambda|}(-1)^{|\Sigma+\Lambda|} C_{|\Sigma+\Lambda|}^{|\Sigma|} d_{\Sigma} v_{r}^{i, \Sigma+\Lambda},
\end{aligned}
$$

is the Noether operator on the fiber bundle (44), coordinated by $\left(x^{\lambda}, y^{i}, \bar{y}_{i}\right)$, with values in the fiber bundle

$$
E^{*} \underset{Y}{\otimes} \wedge T^{*} X
$$

For instance, if a gauge symmetry

$$
v=\left(v_{r}^{i} \xi^{r}+v_{r}^{i, \mu} \xi_{\mu}^{r}+v_{r}^{i, \nu \mu} \xi_{\nu \mu}^{r}\right) \partial_{i}
$$

is of second jet order in parameters, the corresponding Noether operator (54) reads

$$
\Delta_{r}^{i}=v_{r}^{i}-d_{\mu} v_{r}^{i, \mu}+d_{\nu \mu} v_{r}^{i, \nu \mu}, \quad \Delta_{r}^{i, \mu}=-v_{r}^{i, \mu}+2 d_{\nu} v_{r}^{i, \nu \mu}, \quad \Delta_{r}^{i, \nu \mu}=v_{r}^{i, \nu \mu}
$$




\section{Gauge symmetries in gauge gravitation theories}

Turn now to gauge symmetries of gauge gravitation theories mentioned in Section 2.

Let start with the metric-affine gravitation theory on the fiber bundle $\Sigma \times C_{K}$ with the infinitesimal gauge transformations (12) parameterized by vector fields on $X$. In this case, the configuration space (3) of the gauge system is the bundle product

$$
E=\underset{X}{\Sigma} \underset{X}{\times} C_{K} \underset{X}{x}
$$

coordinated by $\left(x^{\lambda}, \sigma^{\mu \nu}, k_{\lambda}{ }^{\nu} \alpha, \tau^{\lambda}\right)$, and the gauge symmetry (4) is the generalized vector field

$$
\begin{aligned}
v= & \left(\tau_{\nu}^{\alpha} \sigma^{\nu \beta}+\tau_{\nu}^{\beta} \sigma^{\alpha \nu}-\tau^{\nu} \sigma_{\nu}^{\alpha \beta}\right) \frac{\partial}{\partial \sigma^{\alpha \beta}}+ \\
& \left(\tau_{\nu}^{\alpha} k_{\mu}{ }^{\nu}{ }_{\beta}-\tau_{\beta}^{\nu} k_{\mu}{ }^{\alpha}{ }_{\nu}-\tau_{\mu}^{\nu} k_{\nu}{ }^{\alpha}{ }_{\beta}+\tau_{\mu \beta}^{\alpha}-\tau^{\nu} k_{\nu \mu}{ }^{\alpha}{ }_{\beta}\right) \frac{\partial}{\partial k_{\mu}{ }^{\alpha} \beta} .
\end{aligned}
$$

Let us consider the gauge theory of principal connections in the presence of a metric gravitational field on the fiber bundle $C \times \Sigma$ with the infinitesimal gauge transformations (16). The configuration space of the corresponding gauge system is the bundle product

$$
E=\underset{X}{\times} \underset{X}{\times} \underset{X}{\times} T_{G} P
$$

coordinated by $\left(x^{\lambda}, a_{\lambda}^{r}, \sigma^{\alpha \beta}, \tau^{\lambda}, \xi^{r}\right)$, and its gauge symmetry is given by the generalized vector field

$$
v=\left(c_{p q}^{r} a_{\lambda}^{p} \xi^{q}+\xi_{\lambda}^{r}-\tau_{\lambda}^{\mu} a_{\mu}^{r}-\tau^{\mu} a_{\mu \lambda}^{r}\right) \partial_{r}^{\lambda}+\left(\tau_{\nu}^{\alpha} \sigma^{\nu \beta}+\tau_{\nu}^{\beta} \sigma^{\alpha \nu}-\tau^{\lambda} \sigma_{\lambda}^{\alpha \beta}\right) \frac{\partial}{\partial \sigma^{\alpha \beta}} .
$$

The configuration space of the gauge theory of gravity in the presence of Dirac fermion

fields is the fiber bundle (37). In the case of infinitesimal gauge transformations (38), the corresponding gauge system is defined on the bundle product

$$
E=\underset{\Sigma_{T}}{\times} C_{K} \underset{\Sigma_{T}}{\times} T X
$$

coordinated by $\left(x^{\lambda}, \sigma_{a}^{\mu}, k_{\lambda}{ }^{\nu} \alpha, y^{A}, \tau^{\lambda}\right)$. Its gauge symmetry reads

$$
\begin{aligned}
v= & \frac{1}{2}\left(\eta^{k d} \eta_{a c} \sigma_{\mu}^{a} \sigma_{d}^{\alpha}+\delta_{\mu}^{\alpha} \delta_{c}^{k}\right)\left(\tau_{\nu}^{\mu} \sigma_{k}^{\nu}-\tau^{\nu} \sigma_{\nu k}^{\mu}\right) \partial_{\alpha}^{c}+ \\
& {\left[\frac{1}{4}\left(\eta^{k b} \sigma_{\mu}^{a}-\eta^{k a} \sigma_{\mu}^{b}\right)\left(\tau_{\nu}^{\mu} \sigma_{k}^{\nu}-\tau^{\nu} \sigma_{\nu k}^{\mu}\right) L_{a b}{ }^{A}{ }_{B} y^{B}-\tau^{\nu} y_{\nu}^{A}\right] \partial_{A}+} \\
& \left(\tau_{\nu}^{\alpha} k_{\mu}{ }^{\nu}{ }_{\beta}-\tau_{\beta}^{\nu} k_{\mu}{ }^{\alpha}{ }_{\nu}-\tau_{\mu}^{\nu} k_{\nu}{ }^{\alpha}{ }_{\beta}+\tau_{\mu \beta}^{\alpha}-\tau^{\nu} k_{\nu \mu}{ }^{\alpha}{ }_{\beta}\right) \frac{\partial}{\partial k_{\mu}{ }^{\alpha}{ }_{\beta}} .
\end{aligned}
$$


If infinitesimal gauge transformations are vector fields (39), the configuration space of this gauge system is the bundle product

$$
E=S \underset{\Sigma_{T}}{\times} C_{K} \underset{\Sigma_{T}}{\times} T_{\mathrm{L}_{s}} P^{0},
$$

coordinated by $\left(x^{\lambda}, \sigma_{a}^{\mu}, k_{\lambda}{ }^{\nu}{ }_{\alpha}, y^{A}, \tau^{\lambda}, \xi^{m}\right)$, and the gauge symmetry is

$$
\begin{aligned}
v= & \left(\tau_{\nu}^{\alpha} \sigma_{c}^{\nu}-\tau^{\nu} \sigma_{\nu c}^{\alpha}-\xi^{m} I_{m}{ }^{d}{ }_{c} \sigma_{d}^{\alpha}\right) \partial_{\alpha}^{c}+\left(\xi^{m} I_{m}{ }^{A}{ }_{B} y^{B}-\tau^{\nu} y_{\nu}^{A}\right) \partial_{A}+ \\
& \left(\tau_{\nu}^{\alpha} k_{\mu}{ }^{\nu}{ }_{\beta}-\tau_{\beta}^{\nu} k_{\mu}{ }^{\alpha}{ }_{\nu}-\tau_{\mu}^{\nu} k_{\nu}{ }^{\alpha}{ }_{\beta}+\tau_{\mu \beta}^{\alpha}-\tau^{\nu} k_{\nu \mu}{ }^{\alpha}{ }_{\beta}\right) \frac{\partial}{\partial k_{\mu}{ }^{\alpha}{ }_{\beta}} .
\end{aligned}
$$

\section{$5 \quad$ BRST symmetries}

In order to introduce BRST symmetries, let us consider Lagrangian systems of even and odd variables. We describe odd variables and their jets on a smooth manifold $X$ as generating elements of the structure ring of a graded manifold whose body is $X[8,29,30]$. This definition reproduces the heuristic notion of jets of ghosts in the field-antifield BRST theory $[31,32]$.

Recall that any graded manifold $(\mathfrak{A}, X)$ with a body $X$ is isomorphic to the one whose structure sheaf $\mathfrak{A}_{Q}$ is formed by germs of sections of the exterior product

$$
\wedge Q^{*}=\mathbb{R} \oplus_{X} Q^{*} \oplus_{X}^{2} \wedge Q^{*} \oplus_{X} \cdots
$$

where $Q^{*}$ is the dual of some real vector bundle $Q \rightarrow X$ of fiber dimension $m$. In field models, a vector bundle $Q$ is usually given from the beginning. Therefore, we consider graded manifolds $\left(X, \mathfrak{A}_{Q}\right)$ where the above mentioned isomorphism holds, and call $\left(X, \mathfrak{A}_{Q}\right)$ the simple graded manifold constructed from $Q$. The structure ring $\mathcal{A}_{Q}$ of sections of $\mathfrak{A}_{Q}$ consists of sections of the exterior bundle (66) called graded functions. Let $\left\{c^{a}\right\}$ be the fiber basis for $Q^{*} \rightarrow X$, together with transition functions $c^{a}=\rho_{b}^{a} c^{b}$. It is called the local basis for the graded manifold $\left(X, \mathfrak{A}_{Q}\right)$. With respect to this basis, graded functions read

$$
f=\sum_{k=0}^{m} \frac{1}{k !} f_{a_{1} \ldots a_{k}} c^{a_{1}} \cdots c^{a_{k}}
$$

where $f_{a_{1} \cdots a_{k}}$ are local smooth real functions on $X$.

Given a graded manifold $\left(X, \mathfrak{A}_{Q}\right)$, let $\mathfrak{d} \mathcal{A}_{Q}$ be the $\mathcal{A}_{Q}$-module of $\mathbb{Z}_{2}$-graded derivations of the $\mathbb{Z}_{2}$-graded ring of $\mathcal{A}_{Q}$, i.e.,

$$
u\left(f f^{\prime}\right)=u(f) f^{\prime}+(-1)^{[u][f]} f u\left(f^{\prime}\right), u \in \mathfrak{d} \mathcal{A}_{Q}, \quad f, f^{\prime} \in \mathcal{A}_{Q},
$$

where [.] denotes the Grassmann parity. Its elements are called $\mathbb{Z}_{2}$-graded (or, simply, graded) vector fields on $\left(X, \mathfrak{A}_{Q}\right)$. Due to the canonical splitting $V Q=Q \times Q$, the vertical 
tangent bundle $V Q \rightarrow Q$ of $Q \rightarrow X$ can be provided with the fiber bases $\left\{\partial_{a}\right\}$ which is the dual of $\left\{c^{a}\right\}$. Then a graded vector field takes the local form $u=u^{\lambda} \partial_{\lambda}+u^{a} \partial_{a}$, where $u^{\lambda}, u^{a}$ are local graded functions. It acts on $\mathcal{A}_{Q}$ by the rule

$$
\left.u\left(f_{a \ldots b} c^{a} \cdots c^{b}\right)=u^{\lambda} \partial_{\lambda}\left(f_{a \ldots b}\right) c^{a} \cdots c^{b}+u^{d} f_{a \ldots b} \partial_{d}\right\rfloor\left(c^{a} \cdots c^{b}\right) .
$$

This rule implies the corresponding transformation law

$$
u^{\prime \lambda}=u^{\lambda}, \quad u^{\prime a}=\rho_{j}^{a} u^{j}+u^{\lambda} \partial_{\lambda}\left(\rho_{j}^{a}\right) c^{j} .
$$

Then one can show that graded vector fields on a simple graded manifold can be represented by sections of the vector bundle $\mathcal{V}_{Q} \rightarrow X$, locally isomorphic to $\wedge Q^{*} \otimes_{X}\left(Q \oplus_{X} T X\right)$.

Accordingly, graded exterior forms on the graded manifold $\left(X, \mathfrak{A}_{Q}\right)$ are introduced as sections of the exterior bundle $\wedge \mathcal{V}_{Q}^{*}$, where $\mathcal{V}_{Q}^{*} \rightarrow X$ is the $\wedge Q^{*}$-dual of $\mathcal{V}_{Q}$. Relative to the dual local bases $\left\{d x^{\lambda}\right\}$ for $T^{*} X$ and $\left\{d c^{b}\right\}$ for $Q^{*}$, graded one-forms read

$$
\phi=\phi_{\lambda} d x^{\lambda}+\phi_{a} d c^{a}, \quad \phi_{a}^{\prime}=\rho_{a}^{-1 b} \phi_{b}, \quad \phi_{\lambda}^{\prime}=\phi_{\lambda}+\rho_{a}^{-1 b} \partial_{\lambda}\left(\rho_{j}^{a}\right) \phi_{b} c^{j} .
$$

The duality morphism is given by the interior product

$$
u\rfloor \phi=u^{\lambda} \phi_{\lambda}+(-1)^{\left[\phi_{a}\right]} u^{a} \phi_{a} .
$$

Graded exterior forms constitute the bigraded differential algebra (henceforth BGDA) $\mathcal{C}_{Q}^{*}$ with respect to the bigraded exterior product $\wedge$ and the exterior differential $d$.

Since the jet bundle $J^{r} Q \rightarrow X$ of a vector bundle $Q \rightarrow X$ is a vector bundle, let us consider the simple graded manifold $\left(X, \mathfrak{A}_{J^{r} Q}\right)$ constructed from $J^{r} Q \rightarrow X$. Its local basis is $\left\{x^{\lambda}, c_{\Lambda}^{a}\right\}, 0 \leq|\Lambda| \leq r$, together with the transition functions

$$
c_{\lambda+\Lambda}^{\prime a}=d_{\lambda}\left(\rho_{j}^{a} c_{\Lambda}^{j}\right), \quad d_{\lambda}=\partial_{\lambda}+\sum_{|\Lambda|<r} c_{\lambda+\Lambda}^{a} \partial_{a}^{\Lambda},
$$

where $\partial_{a}^{\Lambda}$ are the duals of $c_{\Lambda}^{a}$. Let $\mathcal{C}_{J^{r} Q}^{*}$ be the BGDA of graded exterior forms on the graded manifold $\left(X, \mathfrak{A}_{J^{r} Q}\right)$. A linear bundle morphism $\pi_{r-1}^{r}: J^{r} Q \rightarrow J^{r-1} Q$ yields the corresponding monomorphism of BGDAs $\mathcal{C}_{J^{r-1} Q}^{*} \rightarrow \mathcal{C}_{J^{r} Q}^{*}$. Hence, there is the direct system of BGDAs

$$
\mathcal{C}_{Q}^{*} \stackrel{\pi_{0}^{1 *}}{\longrightarrow} \mathcal{C}_{J^{1} Q}^{*} \cdots \stackrel{\pi_{r-1}^{r}}{\longrightarrow} \mathcal{C}_{J^{r} Q}^{*} \longrightarrow \cdots
$$

Its direct limit $\mathcal{C}_{\infty}^{*} Q$ consists of graded exterior forms on graded manifolds $\left(X, \mathfrak{A}_{J^{r} Q}\right), r \in$ $\mathbb{N}$, modulo the pull-back identification, and it inherits the BGDA operations intertwined by the monomorphisms $\pi_{r-1}^{r}{ }^{*}$. It is a $C^{\infty}(X)$-algebra locally generated by the elements $\left(1, c_{\Lambda}^{a}, d x^{\lambda}, \theta_{\Lambda}^{a}=d c_{\Lambda}^{a}-c_{\lambda+\Lambda}^{a} d x^{\lambda}\right), 0 \leq|\Lambda|$.

In order to regard even and odd dynamic variables on the same footing, let $Y \rightarrow X$ be hereafter an affine bundle (or a subbundle of an affine bundle which need not be affine), 
and let $\mathcal{P}_{\infty}^{*} Y \subset \mathcal{O}_{\infty}^{*} Y$ be the $C^{\infty}(X)$-subalgebra of exterior forms whose coefficients are polynomial in the fiber coordinates $y_{\Lambda}^{i}$ on jet bundles $J^{r} Y \rightarrow X$. Let us consider the product

$$
\mathcal{S}_{\infty}^{*}=\mathcal{C}_{\infty}^{*} Q \wedge \mathcal{P}_{\infty}^{*} Y
$$

of graded algebras $\mathcal{C}_{\infty}^{*} Q$ and $\mathcal{P}_{\infty}^{*} Y$ over their common graded subalgebra $\mathcal{O}^{*} X$ of exterior forms on $X$ [29]. It consists of the elements

$$
\sum_{i} \psi_{i} \otimes \phi_{i}, \quad \sum_{i} \phi_{i} \otimes \psi_{i}, \quad \psi \in \mathcal{C}_{\infty}^{*} Q, \quad \phi \in \mathcal{P}_{\infty}^{*} Y
$$

modulo the commutation relations

$$
\begin{aligned}
& \psi \otimes \phi=(-1)^{|\psi||\phi|} \phi \otimes \psi, \quad \psi \in \mathcal{C}_{\infty}^{*} Q, \quad \phi \in \mathcal{P}_{\infty}^{*} Y, \\
& (\psi \wedge \sigma) \otimes \phi=\psi \otimes(\sigma \wedge \phi), \quad \sigma \in \mathcal{O}^{*} X \text {. }
\end{aligned}
$$

They are endowed with the total form degree $|\psi|+|\phi|$ and the total Grassmann parity $[\psi]$. Their multiplication

$$
(\psi \otimes \phi) \wedge\left(\psi^{\prime} \otimes \phi^{\prime}\right):=(-1)^{\left|\psi^{\prime}\right||\phi|}\left(\psi \wedge \psi^{\prime}\right) \otimes\left(\phi \wedge \phi^{\prime}\right)
$$

obeys the relation

$$
\varphi \wedge \varphi^{\prime}=(-1)^{|\varphi|\left|\varphi^{\prime}\right|+[\varphi]\left[\varphi^{\prime}\right]} \varphi^{\prime} \wedge \varphi, \quad \varphi, \varphi^{\prime} \in \mathcal{S}_{\infty}^{*}
$$

and makes $\mathcal{S}_{\infty}^{*}(70)$ into a bigraded $C^{\infty}(X)$-algebra. For instance, elements of the ring $S_{\infty}^{0}$ are polynomials of $c_{\Lambda}^{a}$ and $y_{\Lambda}^{i}$ with coefficients in $C^{\infty}(X)$.

The algebra $\mathcal{S}_{\infty}^{*}$ is provided with the exterior differential

$$
d(\psi \otimes \phi):=\left(d_{\mathcal{C}} \psi\right) \otimes \phi+(-1)^{|\psi|} \psi \otimes\left(d_{\mathcal{P}} \phi\right), \quad \psi \in \mathcal{C}_{\infty}^{*}, \quad \phi \in \mathcal{P}_{\infty}^{*},
$$

where $d_{\mathcal{C}}$ and $d_{\mathcal{P}}$ are exterior differentials on the differential algebras $\mathcal{C}_{\infty}^{*} Q$ and $\mathcal{P}_{\infty}^{*} Y$, respectively. It obeys the relations

$$
d\left(\varphi \wedge \varphi^{\prime}\right)=d \varphi \wedge \varphi^{\prime}+(-1)^{|\varphi|} \varphi \wedge d \varphi^{\prime}, \quad \varphi, \varphi^{\prime} \in \mathcal{S}_{\infty}^{*}
$$

and makes $\mathcal{S}_{\infty}^{*}$ into a BGDA. We agree to call elements of $\mathcal{S}_{\infty}^{*}$ the graded exterior forms on $X$. Hereafter, let the collective symbols $s_{\Lambda}^{A}$ stand both for even and odd generating elements $c_{\Lambda}^{a}, y_{\Lambda}^{i}$ of the $C^{\infty}(X)$-ring $\mathcal{S}_{\infty}^{0}$. Then the BGDA $\mathcal{S}_{\infty}^{*}$ is locally generated by $\left(1, s_{\Lambda}^{A}, d x^{\lambda}, \theta_{\Lambda}^{A}=d s_{\Lambda}^{A}-s_{\lambda+\Lambda}^{A} d x^{\lambda}\right),|\Lambda| \geq 0$. Since the generating elements $s_{\Lambda}^{A}$ and $\theta_{\Lambda}^{A}$ are derived from $s^{A}$, the set $\left\{s^{A}\right\}$ is called the local basis for the BGDA $S_{\infty}^{*}$. 
Similarly to $\mathcal{O}_{\infty}^{*} Y$, the BGDA $\mathcal{S}_{\infty}^{*}$ is decomposed into $\mathcal{S}_{\infty}^{0}$-modules $\mathcal{S}_{\infty}^{k, r}$ of $k$-contact and $r$-horizontal graded forms together with the corresponding projections $h_{k}$ and $h^{r}$. Accordingly, the exterior differential $d(73)$ on $\mathcal{S}_{\infty}^{*}$ is split into the sum $d=d_{H}+d_{V}$ of the total and vertical differentials

$$
d_{H}(\phi)=d x^{\lambda} \wedge d_{\lambda}(\phi), \quad d_{V}(\phi)=\theta_{\Lambda}^{A} \wedge \partial_{A}^{\Lambda} \phi, \quad \phi \in \mathcal{S}_{\infty}^{*} .
$$

One can think of the elements

$$
L=\mathcal{L} \omega \in \mathcal{S}_{\infty}^{0, n}, \quad \delta(L)=\sum_{|\Lambda| \geq 0}(-1)^{|\Lambda|} \theta^{A} \wedge d_{\Lambda}\left(\partial_{A}^{\Lambda} L\right) \in \mathcal{S}_{\infty}^{0, n}
$$

as being a graded Lagrangian and its Euler-Lagrange operator, respectively.

A graded derivation $\vartheta \in \mathfrak{d} \mathcal{S}_{\infty}^{0}$ of the $\mathbb{R}$-ring $\mathcal{S}_{\infty}^{0}$ is said to be contact if the Lie derivative $\mathbf{L}_{\vartheta}$ preserves the ideal of contact graded forms of the BGDA $\mathcal{S}_{\infty}^{*}$. With respect to the local basis $\left(x^{\lambda}, s_{\Lambda}^{A}, d x^{\lambda}, \theta_{\Lambda}^{A}\right)$ for the BGDA $\mathcal{S}_{\infty}^{*}$, any contact graded derivation takes the form

$$
\vartheta=\vartheta_{H}+\vartheta_{V}=\vartheta^{\lambda} d_{\lambda}+\left(\vartheta^{A} \partial_{A}+\sum_{|\Lambda|>0} d_{\Lambda} \vartheta^{A} \partial_{A}^{\Lambda}\right),
$$

where $\vartheta^{\lambda}, \vartheta^{A}$ are local graded functions [29]. The interior product $\left.\vartheta\right\rfloor \phi$ and the Lie derivative $\mathbf{L}_{\vartheta} \phi, \phi \in \mathcal{S}_{\infty}^{*}$, are defined by the formulae

$$
\begin{aligned}
& \vartheta\rfloor \phi=\vartheta^{\lambda} \phi_{\lambda}+(-1)^{\left[\phi_{A}\right]} \vartheta^{A} \phi_{A}, \quad \phi \in \mathcal{S}_{\infty}^{1}, \\
& \left.\vartheta\rfloor(\phi \wedge \sigma)=(\vartheta\rfloor \phi) \wedge \sigma+(-1)^{|\phi|+[\phi][\vartheta]} \phi \wedge(\vartheta\rfloor \sigma\right), \quad \phi, \sigma \in \mathcal{S}_{\infty}^{*}, \\
& \left.\left.\mathbf{L}_{\vartheta} \phi=\vartheta\right\rfloor d \phi+d(\vartheta\rfloor \phi\right), \quad \mathbf{L}_{\vartheta}(\phi \wedge \sigma)=\mathbf{L}_{\vartheta}(\phi) \wedge \sigma+(-1)^{[\vartheta][\phi]} \phi \wedge \mathbf{L}_{\vartheta}(\sigma) .
\end{aligned}
$$

The Lie derivative $\mathbf{L}_{\vartheta} L$ of a Lagrangian $L$ along a contact graded derivation $\vartheta$ (74) fulfills the first variational formula

$$
\left.\left.\left.\mathbf{L}_{\vartheta} L=\vartheta_{V}\right\rfloor \delta L+d_{H}\left(h_{0}(\vartheta\rfloor \Xi_{L}\right)\right)+d_{V}\left(\vartheta_{H}\right\rfloor \omega\right) \mathcal{L},
$$

where $\Xi_{L}$ is a Lepagean equivalent of a graded Lagrangian $L$ [29].

A contact graded derivation $\vartheta$ is said to be variational if the Lie derivative (75) is $d_{H^{-}}$ exact. A glance at the expression (75) shows that: (i) a contact graded derivation $\vartheta$ is variational only if it is projected onto $X$, and (ii) $\vartheta$ is variational iff its vertical part $\vartheta_{V}$ is well. Therefore, we restrict our consideration to vertical contact graded derivations

$$
\vartheta=\sum_{0 \leq|\Lambda|} d_{\Lambda} v^{A} \partial_{A}^{\Lambda} .
$$

Such a derivation is completely defined by its first summand

$$
v=v^{A}\left(x^{\lambda}, s_{\Lambda}^{A}\right) \partial_{A}, \quad 0 \leq|\Lambda| \leq k,
$$


which is also a graded derivation of $\mathcal{S}_{\infty}^{0}$. It is called the generalized graded vector field. A glance at the first variational formula (75) shows that $\vartheta(76)$ is variational iff $v\rfloor \delta L$ is $d_{H^{-}}$exact.

A vertical contact graded derivation $\vartheta(76)$ is said to be nilpotent if

$$
\mathbf{L}_{v}\left(\mathbf{L}_{v} \phi\right)=\sum_{|\Sigma| \geq 0,|\Lambda| \geq 0}\left(v_{\Sigma}^{B} \partial_{B}^{\Sigma}\left(v_{\Lambda}^{A}\right) \partial_{A}^{\Lambda}+(-1)^{\left[s^{B}\right]\left[v^{A}\right]} v_{\Sigma}^{B} v_{\Lambda}^{A} \partial_{B}^{\Sigma} \partial_{A}^{\Lambda}\right) \phi=0
$$

for any horizontal graded form $\phi \in S_{\infty}^{0, *}$ or, equivalently, $(\vartheta \circ \vartheta)(f)=0$ for any graded function $f \in \mathcal{S}_{\infty}^{0}$. One can show that $\vartheta$ is nilpotent only if it is odd and iff the equality

$$
\vartheta\left(v^{A}\right)=\sum_{|\Sigma| \geq 0} v_{\Sigma}^{B} \partial_{B}^{\Sigma}\left(v^{A}\right)=0
$$

holds for all $v^{A}[29]$.

Return now to the original gauge system on a fiber bundle $Y$ with a Lagrangian $L$ (1) and a gauge symmetry $v(4)$. Let us consider the BGDA

$$
\mathcal{S}_{\infty}^{*}[W ; Y]=\mathcal{C}_{\infty}^{*} W \wedge \mathcal{P}_{\infty}^{*} Y
$$

whose local basis is $\left\{c^{r}, y^{i}\right\}$. Let $L \in \mathcal{O}_{\infty}^{0, n} Y$ be a polynomial in $y_{\Lambda}^{i}, 0 \leq|L|$. Then it is a graded Lagrangian $L \in \mathcal{P}_{\infty}^{0, n} Y \subset \mathcal{S}_{\infty}^{0, n}[W ; Y]$ in $\mathcal{S}_{\infty}^{*}[W ; Y]$. Its gauge symmetry $v(4)$ gives rise to the generalized vector field $v_{E}=v$ on $E$, and the latter defines the generalized graded vector field $v(77)$ by the formula (5). It is easily justified that the contact graded derivation $\vartheta(76)$ generated by $v(5)$ is variational for $L$. It is odd, but need not be nilpotent. However, one can try to find a nilpotent contact graded derivation (76) generated by some generalized graded vector field (6) which coincides with $\vartheta$ on $\mathcal{P}_{\infty}^{*} Y$. Then $v(6)$ is called a BRST symmetry.

In this case, the nilpotency conditions (79) read

$$
\begin{aligned}
& \sum_{\Sigma} d_{\Sigma}\left(\sum_{\Xi} v_{r}^{i, \Xi} c_{\Xi}^{r}\right) \sum_{\Lambda} \partial_{i}^{\Sigma}\left(v_{s}^{j, \Lambda}\right) c_{\Lambda}^{s}+\sum_{\Lambda} d_{\Lambda}\left(u^{r}\right) v_{r}^{j, \Lambda}=0, \\
& \sum_{\Lambda}\left(\sum_{\Xi} d_{\Lambda}\left(v_{r}^{i, \Xi} c_{\Xi}^{r}\right) \partial_{i}^{\Lambda}+d_{\Lambda}\left(u^{r}\right) \partial_{r}^{\Lambda}\right) u^{q}=0
\end{aligned}
$$

for all indices $j$ and $q$. They are equations for graded functions $u^{r} \in \mathcal{S}_{\infty}^{0}[W ; Y]$. Since these functions are polynomials

$$
u^{r}=u_{(0)}^{r}+\sum_{\Gamma} u_{(1) p}^{r, \Gamma} c_{\Gamma}^{p}+\sum_{\Gamma_{1}, \Gamma_{2}} u_{(2) p_{1} p_{2}}^{r, \Gamma_{1} \Gamma_{2}} c_{\Gamma_{1}}^{p_{1}} c_{\Gamma_{2}}^{p_{2}}+\cdots
$$

in $c_{\Lambda}^{s}$, the equations $(80)-(81)$ take the form

$$
\sum_{\Sigma} d_{\Sigma}\left(\sum_{\Xi} v_{r}^{i, \Xi} c_{\Xi}^{r}\right) \sum_{\Lambda} \partial_{i}^{\Sigma}\left(v_{s}^{j, \Lambda}\right) c_{\Lambda}^{s}+\sum_{\Lambda} d_{\Lambda}\left(u_{(2)}^{r}\right) v_{r}^{j, \Lambda}=0
$$




$$
\begin{aligned}
& \sum_{\Lambda} d_{\Lambda}\left(u_{(k \neq 2)}^{r}\right) v_{r}^{j, \Lambda}=0, \\
& \sum_{\Lambda} \sum_{\Xi} d_{\Lambda}\left(v_{r}^{i, \Xi} c_{\Xi}^{r}\right) \partial_{i}^{\Lambda} u_{(k-1)}^{q}+\sum_{m+n-1=k} d_{\Lambda}\left(u_{(m)}^{r}\right) \partial_{r}^{\Lambda} u_{(n)}^{q}=0 .
\end{aligned}
$$

One can think of the equalities (83) and (85) as being the generalized commutation relations and generalized Jacobi identities of original gauge transformations, respectively [9].

\section{$6 \quad$ BRST symmetries in gauge gravitation theories}

Following the procedure in Section 5, let us obtain BRST symmetries of gauge gravitation theories listed in Section 2.

Given a gauge system on the fiber bundle (58), let us consider the BGDA

$$
\mathcal{S}_{\infty}^{*}\left[T X ; \sum \underset{X}{\times} C_{K}\right]=\mathcal{C}_{\infty}^{*}[T X] \wedge \mathcal{P}_{\infty}\left[\Sigma \underset{X}{\times} C_{K}\right]
$$

whose basis consists of even elements $\sigma^{\alpha \beta}, k_{\mu}{ }^{\alpha}{ }_{\beta}$ and odd elements $c^{\lambda}$, which are ghosts substituting for parameters $\tau^{\lambda}$ in the gauge symmetry (59). We obtain the nilpotent BRST symmetry

$$
\begin{aligned}
v= & v^{\alpha \beta} \frac{\partial}{\partial \sigma^{\alpha \beta}}+v_{\mu}{ }^{\alpha}{ }_{\beta} \frac{\partial}{\partial k_{\mu}{ }^{\alpha} \beta}+v^{\lambda} \frac{\partial}{\partial c^{\lambda}}=\left(\sigma^{\nu \beta} c_{\nu}^{\alpha}+\sigma^{\alpha \nu} c_{\nu}^{\beta}-c^{\lambda} \sigma_{\lambda}^{\alpha \beta}\right) \frac{\partial}{\partial \sigma^{\alpha \beta}}+ \\
& \left(c_{\nu}^{\alpha} k_{\mu}{ }^{\nu}{ }_{\beta}-c_{\beta}^{\nu} k_{\mu}{ }^{\alpha}{ }_{\nu}-c_{\mu}^{\nu} k_{\nu}{ }^{\alpha}{ }_{\beta}+c_{\mu \beta}^{\alpha}-c^{\lambda} k_{\lambda \mu}{ }^{\alpha}{ }_{\beta}\right) \frac{\partial}{\partial k_{\mu}{ }^{\alpha}{ }_{\beta}}+c_{\mu}^{\lambda} c^{\mu} \frac{\partial}{\partial c^{\lambda}} .
\end{aligned}
$$

The BGDA of the gauge system on the fiber bundle (60) is $\mathcal{S}_{\infty}^{*}\left[T_{G} P ; C \times \Sigma\right]$ whose odd basis elements $c^{r}$ and $c^{\lambda}$ substitute for parameters $\xi^{r}$ and $\tau^{\lambda}$ in the gauge symmetry (61). The corresponding BRST symmetry reads

$$
\begin{aligned}
v= & \left(c_{p q}^{r} a_{\lambda}^{p} c^{q}+c_{\lambda}^{r}-a_{\mu}^{r} c_{\lambda}^{\mu}-c^{\mu} a_{\mu \lambda}^{r}\right) \frac{\partial}{\partial a_{\lambda}^{r}}+\left(\sigma^{\nu \beta} c_{\nu}^{\alpha}+\sigma^{\alpha \nu} c_{\nu}^{\beta}-c^{\lambda} \sigma_{\lambda}^{\alpha \beta}\right) \frac{\partial}{\partial \sigma^{\alpha \beta}}+ \\
& \left(-\frac{1}{2} c_{p q}^{r} c^{p} c^{q}-c^{\mu} c_{\mu}^{r}\right) \frac{\partial}{\partial c^{r}}+c_{\mu}^{\lambda} c^{\mu} \frac{\partial}{\partial c^{\lambda}} .
\end{aligned}
$$

Turn now to the gauge theory of gravity in the presence of fermion field. Since gauge transformations (35) fail to form a closed algebra, one can not associate to them a nilpotent BRST symmetry. Therefore, let us consider the gauge gravitation system on the fiber bundle (64). Its BGDA is

$$
\mathcal{S}_{\infty}^{*}\left[T_{\mathrm{L}_{s}} P^{0} ; S \underset{\Sigma_{T}}{\times} C_{K}\right]
$$


whose odd basis elements $c^{m}$ and $c^{\lambda}$ play the role of ghosts of the gauge symmetry (65). The associated BRST symmetry is

$$
\begin{aligned}
v= & \left(c_{\nu}^{\alpha} \sigma_{c}^{\nu}-c^{\nu} \sigma_{\nu c}^{\alpha}-c^{m} I_{m}{ }^{d}{ }_{c} \sigma_{d}^{\alpha}\right) \frac{\partial}{\partial \sigma_{c}^{\alpha}}+\left(c^{m} I_{m}{ }^{A}{ }_{B} y^{B}-c^{\nu} y_{\nu}^{A}\right) \partial_{A}+ \\
& \left(c_{\nu}^{\alpha} k_{\mu}{ }^{\nu}{ }_{\beta}-c_{\beta}^{\nu} k_{\mu}{ }^{\alpha}{ }_{\nu}-c_{\mu}^{\nu} k_{\nu}{ }^{\alpha}{ }_{\beta}+c_{\mu \beta}^{\alpha}-c^{\lambda} k_{\lambda \mu}{ }^{\alpha}{ }_{\beta}\right) \frac{\partial}{\partial k_{\mu}{ }^{\alpha} \beta}+ \\
& \left(-\frac{1}{2} c_{p q}^{m} c^{p} c^{q}-c^{\mu} c_{\mu}^{m}\right) \frac{\partial}{\partial c^{m}}+c_{\mu}^{\lambda} c^{\mu} \frac{\partial}{\partial c^{\lambda}} .
\end{aligned}
$$

\section{The BV quantization}

As was mentioned above, we restrict our consideration to the BV quantization of the metricaffine gravitation theory. Its original Lagrangian $L_{\mathrm{MA}}$ on the fiber bundle $\Sigma \times C_{K}$ need not be specified, but it possesses the gauge symmetry $v(59)$. It is important that this gauge symmetry is complete and irreducible.

Let us note that any Lagrangian $L$ has gauge symmetries. In particular, there always exist trivial gauge symmetries

$$
v=\sum_{\Lambda} \eta(M)_{r}^{i, \Lambda} \xi_{\Lambda}^{r}, \quad M_{r}^{i, \Lambda}=\sum_{\Sigma} T^{i, j, \Lambda, \Sigma} d_{\Sigma} \mathcal{E}_{j}, \quad T_{r}^{j, i, \Lambda, \Sigma}=-T_{r}^{i, j, \Sigma, \Lambda} .
$$

Furthermore, given a gauge symmetry $v(4)$, let $h$ be a linear differential operator on some vector bundle $E^{\prime} \rightarrow Y$, coordinated by $\left(x^{\lambda}, y^{i}, \xi^{\prime s}\right)$, with values in the vector bundle $E$. Then the composition $v \circ h=v_{s}^{i, \Lambda} \xi_{\Lambda}^{\prime s} \partial_{i}$ is a variational symmetry of the pull-back onto $E^{\prime}$ of a Lagrangian $L$ on $Y$, i.e., a gauge symmetry of $L$. In view of this ambiguity, we agree to say that a gauge symmetry $v(4)$ of a Lagrangian $L$ is complete if a different gauge symmetry $v^{\prime}$ of $L$ factors through $v$ as

$$
v_{0}^{\prime}=v \circ h+T, \quad T \approx 0 .
$$

In the case of a generic metric-affine Lagrangian $L_{\mathrm{MA}}$ on the jet manifold $J^{1}\left(\Sigma \times C_{K}\right)$, the gauge symmetry $v(59)$ is complete. Although there are Lagrangians, e.g., the HilbertEinstein scalar curvature and the Yang-Mills type Lagrangian which admit more wide gauge symmetries. Note that a metric-affine Lagrangian $L_{\mathrm{MA}}$ usually depends on $k_{\mu}{ }_{\beta}$ through the curvature $R_{\lambda \mu}{ }^{\alpha}{ }_{\beta}(8)$. The Euler-Lagrange operator

$$
\delta L_{\mathrm{MA}}=\mathcal{E}_{\alpha \beta} d \sigma^{\alpha \beta} \wedge \omega+\mathcal{E}^{\mu}{ }_{\alpha}{ }^{\beta} d k_{\mu}{ }^{\alpha}{ }_{\beta} \wedge \omega
$$

of a metric-affine Lagrangian takes its values in the fiber bundle

$$
\left(V^{*} \Sigma \underset{X}{\times} V^{*} C_{K}\right) \underset{\Sigma \times C_{K}}{\otimes} \stackrel{4}{\wedge} T^{*} X
$$


coordinated by $\left(x^{\lambda}, \sigma^{\mu \nu}, k_{\lambda}{ }^{\nu}{ }_{\alpha}, \bar{\sigma}_{\mu \nu}, \bar{k}_{\nu}^{\lambda}{ }_{\nu}^{\alpha}\right)$. Due to the canonical splittings

$$
V^{*} \Sigma=\Sigma \underset{X}{\times} \Sigma^{*}=\Sigma \underset{X}{\times} \stackrel{2}{V}^{2} T X, \quad V^{*} C_{K}=C_{K} \underset{X}{\times} C_{K}^{*}=C_{K} \underset{X}{\times}\left(\underset{\otimes}{\otimes} T \underset{X}{\otimes} T^{*} X\right),
$$

the fiber bundle (91) is isomorphic to

$$
\left(\Sigma \underset{X}{\times} C_{K}\right) \underset{X}{\times}\left(\Sigma^{*} \underset{X}{\times} C_{K}^{*}\right) \underset{X}{\otimes} \stackrel{4}{\wedge} T^{*} X
$$

A complete gauge symmetry $v \not \approx 0(4)$ is called reducible if there exist a vector bundle $W_{0} \rightarrow X$ and a $E$-valued differential operator $v^{0} \not \approx 0$ on the bundle product $E_{0}=Y \times W_{0}$ which is linear on $W_{0}$ and obeys the equality $v \circ v^{0} \approx 0$. If $v^{\prime 0}$ is another differential operator possessing these properties, then $v^{\prime 0}$ factors through $v^{0}$.

The gauge symmetry (59) is irreducible because its term of maximal jet order is $\tau_{\mu \beta}^{\alpha} \partial_{\alpha}^{\mu \beta}$. Indeed, let us assume that there exist a vector bundle $W_{0} \rightarrow X$ coordinated by $\left(x^{\lambda}, w^{A}\right)$ and the above mentioned differential operator $v^{0}$ on the bundle product

$$
E_{0}=\underset{X}{\times} C_{K} \underset{X}{\times} W_{0}
$$

with values in the vector bundle $E(58)$ such that $v \circ v^{0} \approx 0$. Given the maximal jet order term

$$
\sum_{|\Xi|=k} v_{0}^{\alpha, \Xi} w_{\Xi}^{A} \frac{\partial}{\partial \tau^{\alpha}} \not \approx 0
$$

of the operator $v^{0}$, the equality $v \circ v^{0} \approx 0$ results in the condition

$$
\sum_{|\Xi|=k} v_{0 A}^{\alpha, \Xi} d_{\mu \beta} w_{\Xi}^{A} \frac{\partial}{\partial k_{\mu}^{\alpha}{ }^{\alpha}} \approx 0 .
$$

It follows that all coefficients $v_{0 A}^{\alpha, \Xi}$ and, consequently, the term (93) vanish on-shell.

By virtue of Noether's second theorem, the gauge symmetry (59) defines the Noether operator $\Delta$ (54) on the fiber bundle (91) with values in the fiber bundle

$$
\left(\Sigma \underset{X}{\times} C_{K} \underset{X}{\times} T^{*} X\right) \underset{\Sigma \times C_{K}}{\otimes} \stackrel{4}{\wedge} T^{*} X
$$

coordinated by $\left(x^{\lambda}, \sigma^{\mu \nu}, k_{\lambda}{ }^{\nu}{ }_{\alpha}, \bar{\tau}_{\lambda}\right)$. The formula (57) gives

$$
\begin{aligned}
\Delta & =\tau^{\lambda} \sum_{|\Lambda|=0,1,2}\left[\Delta_{\lambda}^{\alpha \beta, \Lambda} d_{\Lambda} \bar{\sigma}_{\alpha \beta}+\Delta_{\lambda \mu}{ }^{\alpha}{ }_{\beta}{ }^{\Lambda}{ }^{\prime} d_{\Lambda} \bar{k}_{\alpha}^{\mu}{ }_{\alpha}^{\beta}\right] \omega= \\
& \tau^{\lambda}\left[-\left(\sigma_{\lambda}^{\alpha \beta}+2 \sigma_{\nu}^{\nu \beta} \delta_{\lambda}^{\alpha}\right) \bar{\sigma}_{\alpha \beta}-2 \sigma^{\nu \beta} d_{\nu} \bar{\sigma}_{\lambda \beta}+\left(-k_{\lambda \mu}{ }^{\alpha}{ }_{\beta}-k_{\nu \mu}{ }_{\beta}{ }_{\beta} \delta_{\lambda}^{\alpha}+k_{\beta \mu}{ }^{\alpha}{ }_{\lambda}+k_{\mu \lambda}{ }_{\beta}\right) \bar{k}_{\alpha}^{\mu}{ }_{\alpha}{ }^{\beta}+\right. \\
& \left.\left(-k_{\mu}{ }^{\nu}{ }_{\beta} \delta_{\lambda}^{\alpha}+k_{\mu}{ }^{\alpha}{ }_{\lambda} \delta_{\beta}^{\nu}+k_{\lambda}{ }^{\alpha}{ }_{\beta} \delta_{\mu}^{\nu}\right) d_{\nu} \bar{k}^{\mu}{ }_{\alpha}{ }^{\beta}+d_{\mu \beta} \bar{k}_{\lambda}^{\mu}{ }_{\lambda}{ }^{\beta}\right] \omega .
\end{aligned}
$$


Following the standard BV quantization procedure $[4,5]$, let us construct the extended Lagrangian which obey certain condition and depends on ghosts and antifields.

In order to introduce antifields of even fields $\sigma^{\alpha \beta}, k_{\mu}{ }^{\alpha}{ }_{\beta}$ and odd ghosts $\tau^{\lambda}$, let us extend the BGDA (86) to the BGDA

$$
\mathcal{S}_{\infty}^{*}\left[T \underset{X}{X} \underset{X}{\times}\left(\Sigma^{*} \underset{X}{\times} C_{K}^{*}\right) \underset{X}{\otimes} \stackrel{4}{\wedge} T^{*} X ; \underset{X}{\times} C_{K} \underset{X}{\times} T^{*} X \underset{X}{\otimes} \stackrel{4}{\wedge} T^{*} X\right]
$$

where the splittings (92) has been used. The local basis for the BGDA (96) is

$$
\left\{\sigma^{\mu \nu}, k_{\mu \beta}^{\alpha}, c^{\lambda}, \bar{\sigma}_{\mu \nu}, \bar{k}_{\alpha}^{\mu}{ }_{\alpha}^{\beta}, \bar{c}_{\lambda}\right\}
$$

where the antifields $\bar{\sigma}_{\mu \nu}, \bar{k}_{\nu}^{\lambda}{ }^{\alpha}$ are odd, while the antifields $\bar{c}_{\lambda}$ are even. It is convenient to use right graded derivations $\overleftarrow{\partial}$ with respect to antifields. They act on graded functions and forms $\phi$ on the right by the rule

$$
\overleftarrow{\partial}(\phi)=d \phi L \overleftarrow{\partial}+d\left(\phi\lfloor\overleftarrow{\partial}), \quad \overleftarrow{\partial}\left(\phi \wedge \phi^{\prime}\right)=(-1)^{\left[\phi^{\prime}\right]} \overleftarrow{\partial}(\phi) \wedge \phi^{\prime}+\phi \wedge \overleftarrow{\partial}\left(\phi^{\prime}\right)\right.
$$

An original metric-affine Lagrangian $L_{\mathrm{MA}}$ is assumed to be polynomial in variables $\sigma^{\mu \nu}$, $k_{\lambda}{ }^{\nu}{ }_{\alpha}$. Therefore, it is obviously a graded Lagrangian in the BGDA (96). Given the BRST symmetry $v(87)$, let us consider the graded density

$$
L=\mathcal{L} \omega=L_{\mathrm{MA}}+v^{\alpha \beta} \bar{\sigma}_{\alpha \beta} \omega+v_{\mu}{ }^{\alpha}{ }_{\beta} \bar{k}_{\alpha}^{\mu}{ }_{\alpha} \omega+v^{\lambda} \bar{c}_{\lambda} \omega .
$$

One can show that it obeys the following conditions

$$
\begin{aligned}
& v_{c}^{\alpha}=\frac{\overleftarrow{\partial} \mathcal{L}}{\partial \bar{\sigma}_{\alpha}^{c}}, \quad v_{\mu}{ }^{\alpha}{ }_{\beta}=\frac{\overleftarrow{\partial} \mathcal{L}}{\partial \bar{k}^{\mu}{ }_{\alpha}^{\beta}}, \quad v^{\lambda}=\frac{\overleftarrow{\partial} \mathcal{L}}{\partial \bar{c}_{\lambda}} \\
& \mathbf{L}_{v} L=\frac{\overleftarrow{\partial} \mathcal{L}}{\partial \bar{\sigma}_{\alpha}^{c}} \frac{\delta L}{\delta \sigma_{c}^{\alpha}}+\frac{\overleftarrow{\partial} \mathcal{L}}{\partial \bar{k}^{\mu}{ }_{\alpha}^{\beta}} \frac{\delta L}{\delta k_{\mu}{ }^{\alpha} \beta}+\frac{\overleftarrow{\partial} \mathcal{L}}{\partial \bar{c}_{\lambda}} \frac{\delta L}{\delta c^{\lambda}}+d_{H} \sigma=d_{H} \sigma^{\prime} \\
& \left.L\right|_{\bar{\sigma}=\bar{k}=\bar{c}=0}=L_{\mathrm{MA}} .
\end{aligned}
$$

A glance at these conditions shows that $L(98)$ is a proper solution of the master equation (100) $[4,5]$. Thus, it is the desired extended Lagrangian.

One can write different variants of a gauged-fixed Lagrangian (see, e.g., [33] for the problem of the general covariance in quantum gravity). By analogy with the Lorenz gauge in Yang-Mills gauge theory, let us choose the gauge-fixing quantity

$$
\mathcal{S}_{\beta}^{\alpha}=\sigma^{\lambda \mu} S_{\lambda \mu \beta}{ }^{\alpha}{ }^{2}=\sigma^{\lambda \mu}\left(k_{\lambda \mu}{ }^{\alpha}{ }_{\beta}+k_{\mu \lambda}{ }^{\alpha}{ }_{\beta}\right),
$$

where $S_{\lambda \mu}{ }^{\alpha}{ }_{\beta}$ is given by the expression (9). In order to define a gauge-fixing density, we add even elements $B_{\beta}^{\alpha}$, odd elements $C_{\alpha}^{\beta}$ and their even antifields $\bar{C}_{\beta}^{\alpha}$ to the local basis for the BGDA (96). Then this gauge-fixing density reads

$$
\Phi=C_{\alpha}^{\beta}\left(\frac{1}{2} B_{\beta}^{\alpha}-\mathcal{S}_{\beta}^{\alpha}\right) \omega .
$$


The desired gauged-fixed Lagrangian $L_{\mathrm{GF}}$ comes from the graded Lagrangian

$$
L+B_{\beta}^{\alpha} \bar{C}_{\alpha}^{\beta} \omega=L_{\mathrm{MA}}+v^{\alpha \beta} \bar{\sigma}_{\alpha \beta} \omega+v_{\mu}{ }_{\beta}{ }_{\beta} \bar{k}_{\alpha}^{\mu}{ }_{\alpha} \omega+v^{\lambda} \bar{c}_{\lambda} \omega+B_{\beta}^{\alpha} \bar{C}_{\alpha}^{\beta} \omega
$$

by means of replacement of antifields with the variational derivatives

$$
\begin{aligned}
& \bar{\sigma}_{\alpha \beta}=\frac{\delta \Phi}{\delta \sigma^{\alpha \beta}}=-C_{\nu}^{\mu}\left(k_{\alpha \beta^{\nu}}{ }_{\mu}+k_{\beta \alpha}{ }^{\nu}{ }_{\mu}\right), \quad \bar{k}^{\mu}{ }^{\beta}{ }^{\beta}=\frac{\delta \Phi}{\delta k_{\mu}{ }^{\alpha} \beta}=2 d_{\lambda}\left(C_{\alpha}^{\beta} \sigma^{\lambda \mu}\right), \\
& \bar{c}_{\lambda}=\frac{\delta \Phi}{\delta c^{\lambda}}=0, \quad \bar{C}_{\alpha}^{\beta}=\frac{\delta \Phi}{\delta C_{\beta}^{\alpha}}=\left(\frac{1}{2} B_{\alpha}^{\beta}-S_{\alpha}^{\beta}\right) .
\end{aligned}
$$

One obtains

$$
\begin{aligned}
\mathcal{L}_{\mathrm{GF}} & =\mathcal{L}_{\mathrm{MA}}-v^{\alpha \beta} C_{\nu}^{\mu}\left(k_{\alpha \beta}{ }^{\nu}{ }_{\mu}+k_{\beta \alpha{ }^{\nu}{ }_{\mu}}\right)+2 v^{\mu}{ }_{\alpha}{ }^{\beta} d_{\gamma}\left(C_{\alpha}^{\beta} \sigma^{\gamma \mu}\right)+B_{\beta}^{\alpha}\left(\frac{1}{2} B_{\alpha}^{\beta}-S_{\alpha}^{\beta}\right)= \\
& \mathcal{L}_{\mathrm{MA}}-\left(\sigma^{\lambda \beta} c_{\lambda}^{\alpha}+\sigma^{\alpha \lambda} c_{\lambda}^{\beta}-c^{\lambda} \sigma_{\lambda}^{\alpha \beta}\right) C_{\nu}^{\mu}\left(k_{\alpha \beta}{ }^{\nu}{ }_{\mu}+k_{\beta \alpha}{ }^{\nu}{ }_{\mu}\right)+ \\
& 2\left(c_{\nu}^{\alpha} k_{\mu}{ }^{\nu}{ }_{\beta}-c_{\beta}^{\nu} k_{\mu}{ }^{\alpha}{ }_{\nu}-c_{\mu}^{\nu} k_{\nu}{ }^{\alpha}{ }_{\beta}+c_{\mu \beta}^{\alpha}-c^{\lambda} k_{\lambda \mu}{ }^{\alpha}{ }_{\beta}\right) d_{\gamma}\left(C_{\alpha}^{\beta} \sigma^{\gamma \mu}\right)+ \\
& B_{\beta}^{\alpha}\left(\frac{1}{2} B_{\alpha}^{\beta}-\sigma^{\lambda \mu}\left(k_{\lambda \mu}{ }^{\beta}{ }_{\alpha}{ }+k_{\mu \lambda}{ }^{\beta}{ }_{\alpha}\right)\right) .
\end{aligned}
$$

Given the Lagrangian (104), one can not automatically write the generating functional of quantum gravity because the BV quantization procedure fails to provide a functional measure. However, this generating functional at least is a Gaussian integral with respect to variables $B_{\beta}^{\alpha}$. Therefore, we can replace the Lagrangian (104) with the effective one which takes the familiar form

$$
\mathcal{L}_{\mathrm{EGF}}=\mathcal{L}_{\mathrm{MA}}+C_{\beta}^{\alpha} \mathcal{M}_{\alpha \lambda}^{\beta} c^{\lambda}+\frac{1}{2} \sigma^{\lambda \mu} \sigma^{\gamma \nu}\left(k_{\lambda \mu}{ }^{\beta}{ }_{\alpha}+k_{\mu \lambda}{ }^{\beta}{ }_{\alpha}\right)\left(k_{\gamma \nu}{ }^{\alpha}{ }+k_{\nu \gamma}{ }^{\alpha}\right),
$$

where $\mathcal{M}$ is a linear third order differential operator acting on ghosts $c^{\lambda}$.

\section{References}

[1] D.Ivanenko and G.Sardanashvily, The gauge treatment of gravity, Phys. Rep. 94 (1983) 1.

[2] F.Hehl, J.McCrea, E.Mielke and Y.Ne'eman, Metric-affine gauge theory of gravity: field equations, Noether identities, world spinors, and breaking of dilaton invariance, Phys. Rep. 258 (1995) 1.

[3] G.Sardanashvily, Classical gauge theory of gravity, Theor. Math. Phys. 132 (2002) 1163; E-print arXiv: gr-qc/0208054.

[4] I.Batalin and G.Vilkoviski, Closure of the gauge algebra, generalized Lie algebra equations and Feynman rules, Nucl. Phys. B234 (1984) 106. 
[5] J.Gomis, J.París and S.Samuel, Antibracket, antifields and gauge theory quantization, Phys. Rep 295 (1995) 1.

[6] L.Mangiarotti and G.Sardanashvily, Connections in Classical and Quantum Field Theory (World Scientific, Singapore, 2000).

[7] G.Sardanashvily, Ten lectures on jet manifolds in classical and quantum field theory, E-print arXiv: math-ph/0203040.

[8] D.Bashkirov, G.Giachetta, L.Mangiarotti and G.Sardanashvily, Noether's second theorem for BRST symmetries, J. Math. Phys. (accepted); E-print arXiv: math$\mathrm{ph} / 0412034$.

[9] G.Sardanashvily, On algebras of gauge transformations in a general setting, E-print arXiv: math.QA/0411635.

[10] N.Steenrod, The Topology of Fibre Bundles (Princeton Univ. Press, Princeton, 1972).

[11] L.Fatibene and M.Francaviglia, Natural and Gauge Natural Formalism for Classical Field Theories. A Geometric Perspective Including Spinors and Gauge Theories (Kluwer, Dordrecht, 2003).

[12] L.Fatibene, M.Ferraris and M.Francaviglia, On the gauge natural structure of modern physics, Int. J. Geom. Methods Mod. Phys. 1 (2004) 443.

[13] G.Giachetta, L.Mangiarotti and G.Sardanashvily, Noether conservation laws in higherdimensional Chern-Simons theory, Mod. Phys. Lett. A 18 (2003) 2645.

[14] J.Crawford, Clifford algebra: Notes on the spinor metric and Lorentz, Poincaré and conformal groups, J. Math.Phys. 32 (1991) 576.

[15] Yu.Obukhov and S.Solodukhin, Dirac equation and the Ivanenko-Landau-Kähler equation, Int. J. Theor. Phys. 33 (1994) 225.

[16] Y.Ne'eman and Dj.Šijački, Unified affine gauge theory of gravity and strong interactions with finite and infinite $\overline{G L}(4, \mathbb{R})$ spinor fields, Ann. Phys. 120 (1979) 292.

[17] L.Dabrowski and R.Percacci, Spinors and diffeomorphisms, Comm. Math. Phys. 106 (1986) 691.

[18] H.Lawson and M-L. Michelson, Spin Geometry (Princeton Univ. Press, Princeton, 1989). 
[19] S.Switt, Natural bundles. II. Spin and the diffeomorphism group, J. Math. Phys. 34 (1993) 3825.

[20] R.Fulp, J.Lawson and L.Norris, Geometric prequantization on the spin bundle based on $n$-symplectic geometry: the Dirac equation, Int. J. Theor. Phys. 33 (1994) 1011.

[21] G.Sardanashvily, Covariant spin structure, J. Math. Phys. 39 (1998) 4874.

[22] R.Geroch, Spinor structure of space-time in general relativity, J. Math. Phys. 9 (1968) 104.

[23] E.Stiefel, Richtungsfelder und fernparallelismus in mannigfaltigkeiten, Comm. Math. Helv. 8 (1936) 3.

[24] S.Avis and C.Isham, Generalized spin structure on four dimensional space-times, Comm. Math. Phys. 72 (1980) 103.

[25] V.Ponomarev and Yu.Obukhov, Generalized Einstein-Maxwell theory, Gen. Rel. Grav. 14 (1982) 309.

[26] G.Sardanashvily, Stress-energy-momentum conservation law in gauge gravitation theory, Class. Quant. Grav. 14 (1997) 1371.

[27] Y.Kosmann, Dérivées de Lie des spineurs, Ann. di Matem. Pura ed Appl. 91 (1972) 317.

[28] L.Fatibene, M.Ferraris, M.Francaviglia and M.Godina, Gauge formalism for General Relativity and fermionic matter, Gen. Rel. Grav. 30 (1998) 1371.

[29] G.Giachetta, L.Mangiarotti and G.Sardanashvily, Lagrangian supersymmetries depending on derivatives. Global analysis and cohomology, Commun. Math. Phys. (accepted); E-print arXiv: hep-th/0407185.

[30] G.Sardanashvily, SUSY-extended field theory, Int. J. Mod. Phys. A 15 (2000) 3095.

[31] G.Barnich, F.Brandt and M.Henneaux, Local BRST cohomology in gauge theories, Phys. Rep. 338 (2000) 439.

[32] F.Brandt, Jet coordinates for local BRST cohomology, Lett. Math. Phys. 55 (2001) 149.

[33] K.Kazakov, General covariance in quantum gravity, Class. Quant. Grav. 19 (2002) 3017. 